\title{
Sex-dependent Effects of Developmental Hypoxia on Cardiac Mitochondria from Adult Murine Offspring
}

DOI:

10.1016/j.freeradbiomed.2020.11.004

\section{Document Version}

Accepted author manuscript

Link to publication record in Manchester Research Explorer

\section{Citation for published version (APA):}

Hellgren, K., Premanandhan, H., Quinn, C., Trafford, A., \& Galli, G. (2020). Sex-dependent Effects of Developmental Hypoxia on Cardiac Mitochondria from Adult Murine Offspring. Free Radical Biology and Medicine. https://doi.org/10.1016/j.freeradbiomed.2020.11.004

\section{Published in:}

Free Radical Biology and Medicine

\section{Citing this paper}

Please note that where the full-text provided on Manchester Research Explorer is the Author Accepted Manuscript or Proof version this may differ from the final Published version. If citing, it is advised that you check and use the publisher's definitive version.

\section{General rights}

Copyright and moral rights for the publications made accessible in the Research Explorer are retained by the authors and/or other copyright owners and it is a condition of accessing publications that users recognise and abide by the legal requirements associated with these rights.

\section{Takedown policy}

If you believe that this document breaches copyright please refer to the University of Manchester's Takedown Procedures [http://man.ac.uk/04Y6Bo] or contact uml.scholarlycommunications@manchester.ac.uk providing relevant details, so we can investigate your claim.

\section{OPEN ACCESS}


1 Sex-dependent Effects of Developmental Hypoxia on Cardiac Mitochondria from Adult Murine Offspring

4 Kim T. Hellgren ${ }^{1}$, Hajani Premanandhan ${ }^{1}$, Callum J. Quinnn ${ }^{1}$, Andrew W Trafford ${ }^{1}$

5 and Gina L. J. Galli ${ }^{* 1}$

6

7 'Division of Cardiovascular Sciences, School of Medical Sciences, University of

8 Manchester, Manchester M13 9NT, UK

9

$10{ }^{*}$ Corresponding Author

11 Dr Gina Galli

12 Division of Cardiovascular Sciences,

13 School of Medical Sciences,

14 University of Manchester,

15 3.09 Core Technology Facility,

1646 Grafton St,

17 Manchester M13 9NT, UK

18 gina.galli@manchester.ac.uk 


\section{Abstract}

20 Insufficient oxygen supply (hypoxia) during fetal and embryonic development can lead to

21 latent phenotypical changes in the adult cardiovascular system, including altered cardiac

22 function and increased susceptibility to ischemia reperfusion injury. While the cellular

23 mechanisms underlying this phenomenon are largely unknown, several studies have

24 pointed towards metabolic disturbances in the heart of offspring from hypoxic

25 pregnancies. To this end, we investigated mitochondrial function in the offspring of a

26 mouse model of prenatal hypoxia. Pregnant C57 mice were subjected to either normoxia

$27(21 \%)$ or hypoxia (14\%) during gestational days 6-18. Offspring were reared in normoxia

28 for up to 8 months and mitochondrial biology was assessed with electron microscopy

29 (ultrastructure), spectrophotometry (enzymatic activity of electron transport chain

30 complexes), microrespirometry (oxidative phosphorylation and $\mathrm{H}_{2} \mathrm{O}_{2}$ production) and

31 Western Blot (protein expression). Our data showed that male adult offspring from

32 hypoxic pregnancies possessed mitochondria with increased $\mathrm{H}_{2} \mathrm{O}_{2}$ production and lower

33 respiratory capacity that was associated with reduced protein expression of complex I, II

34 and IV. In contrast, females from hypoxic pregnancies had a higher respiratory capacity

35 and lower $\mathrm{H}_{2} \mathrm{O}_{2}$ production that was associated with increased enzymatic activity of

36 complex IV. From these results, we speculate that early exposure to hypoxia has long

37 term, sex-dependent effects on cardiac metabolic function, which may have implications

38 for cardiovascular health and disease in adulthood. 


\section{Graphical Abstract}

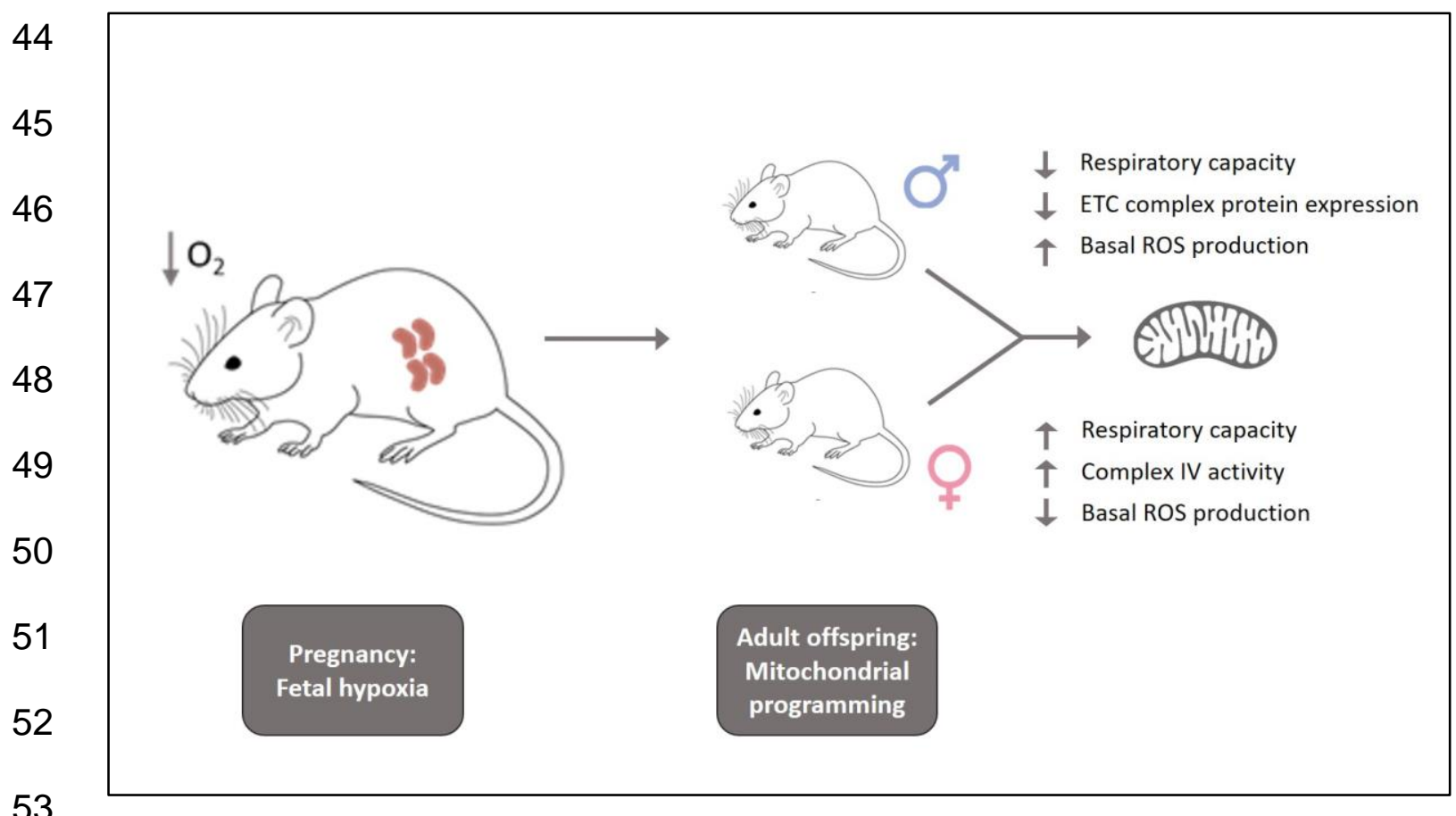

54

55

56 Keywords

57 Heart

58 Mitochondria

59 Developmental programming

60 Fetal hypoxia

61 Reactive oxygen species

62

63

64

65

66 


\section{Abbreviations}

68 ADP - adenosine diphosphate

69 CS - citrate synthase

70 ETC - electron transport chain

$71 \quad \mathrm{ET}_{\mathrm{Cl}+\mathrm{CII}}$ - electron transfer capacity with complex I and II substrates

$72 \mathrm{ET}_{\mathrm{CII}}$ - electron transfer capacity with complex II substrates

73 FCCP - carbonyl cyanide-4-(trifluoromethoxy)phenylhydrazone

74 GD - gestational day

$75 \mathrm{H}_{2} \mathrm{O}_{2}$ - hydrogen peroxide

76 HRP - horseradish peroxidase

$77 \quad \mathrm{I} / \mathrm{R}-$ ischemia reperfusion

$78 \quad$ LEAK $_{\mathrm{Cl}}$ - Leak respiratory state with complex I substrates

79 MPG - malate pyruvate and glutamate

$80 \mathrm{O}_{2}^{-}$- superoxide

81 OXPHOS - oxidative phosphorylation

82 OXPHOS $_{\mathrm{Cl}}$ - oxidative phosphorylation with complex I substrates

$83 \mathrm{OXPHOS} \mathrm{Cl}+\mathrm{Cll}$ - oxidative phosphorylation with complex I and II substrates

84 PKC $\varepsilon$ - protein kinase $\mathrm{C}$ epsilon

$85 \mathrm{RCR}$ - respiratory control ratio

86 ROS - reactive oxygen species

87 ROX - residual non-mitochondrial oxygen consumption

88 SOD - superoxide dismutase

89 TMPD - TM/AS, N,N,N,N-tetramethyl-p-phenylenediamin

90 


\section{1.0. Introduction}

92 Heart disease remains the leading cause of death worldwide ${ }^{1}$. Despite significant

93 advances in treatment options, prevention strategies are the most cost-effective way to

94 reduce the socioeconomic burden of these diseases. Such strategies have traditionally

95 targeted behavioral and lifestyle risk factors, such as smoking and obesity. However,

96 the seminal work of Barker ${ }^{2}$ demonstrated that adverse events during pregnancy can

97 predispose offspring to heart disease in adulthood. This phenomenon, termed

98 developmental programming, provides a window of opportunity to prevent the

99 development of heart disease in early life. Nevertheless, before effective treatments can

100 be designed, it is crucial to understand the mechanisms leading to cardiac dysfunction in

101 offspring from high-risk pregnancies.

102

103 Insufficient oxygen supply to an embryo or foetus, termed developmental hypoxia,

104 occurs in a wide range of high-risk pregnancies, including preeclampsia, placental

105 insufficiency, placental infection, maternal anaemia, gestational diabetes and high

106 altitude pregnancy ${ }^{3,4}$. Animal models have shown the foetus initially responds to

107 hypoxia by preferentially distributing blood flow to vital organs, such as the heart and

108 brain ${ }^{4-6}$. While this strategy is protective in the short term, a sustained redistribution of

109 blood flow is associated with asymmetric fetal growth restriction, increased peripheral

110 resistance and cardiac abnormalities, including hypertrophy ${ }^{4}$. In adulthood, the hearts

111 of offspring from hypoxic pregnancies continue to express abnormal phenotypes,

112 including diastolic dysfunction, sustained increases in myocardial contractility and

113 enhanced responsiveness to $\beta$-adrenoreceptor stimulation ${ }^{7-9}$. Additionally, prenatal

114 hypoxia appears to sensitise the adult heart to ischaemia and reperfusion $(\mathrm{l} / \mathrm{R})$ injury ${ }^{10}$ -

115 12. Interestingly, females from hypoxic pregnancies appear to be partially protected 
116 from cardiac dysfunction, suggesting that the effects of developmental hypoxia are

117 gender-specific ${ }^{11,13,14}$. In aggregate, these studies suggest developmental hypoxia

118 programmes a dysfunctional cardiac phenotype in offspring that cannot be reversed by

119 normalising oxygen availability after birth.

121 Given that most of these experiments have been conducted in isolated hearts, the

122 cardiac dysfunction in offspring from hypoxic pregnancies cannot be explained by

123 autonomic influences, altered peripheral resistance (cardiac afterload) or circulating

124 catecholamines. Therefore, the intrinsic properties of the myocardial cells have been

125 altered. While multiple cellular mechanisms may account for cardiac dysfunction, recent

126 evidence suggests intrauterine stress can alter offspring cardiac metabolism, particularly

127 at the level of the mitochondria. For example, nutritional stress during development

128 leads to a range of cardiac mitochondrial abnormalities in fetal and adult offspring,

129 including structural disorganization, impaired mitophagy, reduced oxygen consumption,

130 decreased proton leak and altered fission/fusion dynamics ${ }^{15-17}$. Similarly, exposure to

131 excess glucocorticoids during development can programme cardiac mitochondrial

132 dysfunction in adult offspring, leading to increased sensitivity to ischemia/reperfusion

133 injury, higher levels of $\mathrm{H}_{2} \mathrm{O}_{2}$ production and a reduced capacity to produce ATP ${ }^{18}$.

134 Collectively, these studies suggest mitochondrial remodeling represents a major

135 mechanism underlying the developmental programming of heart disease.

137 To our knowledge, only one group has investigated the long-term effects of 138 developmental hypoxia on offspring mitochondrial function. Thompson and colleagues

139 has shown pregnant guinea pigs exposed to $10.5 \%$ atmospheric oxygen between 140 gestational days (GD) 28-65 (term 65-72 days) led to sex-specific alterations in 
141 mitochondrial enzymatic complex activities, mitochondrial DNA content, protein

142 expression and respiration ${ }^{19-22}$. However, the moderate level of hypoxia used in these

143 studies (10.5\%) caused a significant reduction in maternal weight, which is indicative of

144 nutritional stress. It is therefore difficult to conclude whether mitochondrial function is

145 being altered by developmental hypoxia or nutritional stress, or a combination of the two.

146 To this end, we have undertaken a comprehensive investigation into the effects of

147 developmental hypoxia on adult offspring mitochondrial morphology, respiratory

148 capacity, reactive oxygen species production, enzymatic activity and protein expression.

149 Our results suggest developmental hypoxia has long-term, sex-dependent effects on

150 cardiac mitochondrial function; in particular, males from hypoxic pregnancies have a

151 lower mitochondrial respiratory capacity and generate more $\mathrm{H}_{2} \mathrm{O}_{2}$ under basal conditions,

152 and females from hypoxic pregnancies have greater respiratory scope and produce less

$153 \mathrm{H}_{2} \mathrm{O}_{2}$ under basal conditions, compared to their normoxic counterparts. We speculate

154 that these phenotypes have long-term implications for metabolic health and the

155 susceptibility to heart disease.

157 2.0. Methods

158 2.1. Animal model

159 There are several strategies to model chronic fetal hypoxia, but most (e.g. placental 160 embolisation, reduced uterine blood flow) cause impaired placental perfusion, thereby 161 decreasing the delivery of nutrients as well as oxygen to the foetus ${ }^{23,24}$. In these 162 instances, it is difficult to separate the effects of fetal nutrient restriction versus fetal 163 hypoxia in programming cardiovascular dysfunction in the offspring. To this end, we 164 have utilised a rodent model of prenatal hypoxia developed by the Giussani laboratory 
165 that does not affect maternal food intake, thereby allowing the effects of developmental

166 hypoxia to be studied in isolation ${ }^{8,25-27}$.

168 2.2. Oxygen incubation protocols

169 All procedures comply with The UK Animals (Scientific Procedures) Act 1986 and EU

170 directive 2010/63. The ARRIVE guidelines were followed for reporting the use of animals

171 in scientific experiments. Local ethical approval was granted by The University of

172 Manchester Animal Welfare Ethical and Review Board. Pregnant C57BL/J6 mice (aged

17312 weeks, $24.1 \pm 6.0 \mathrm{~g}$ ), were bred, mated and maintained at The University of

174 Manchester (UK). Mice were housed in standard Individually Ventilated Cages (IVC)

175 with normal oxygen levels and a 12:12 light cycle with ad libitum food and water. The

176 pregnant mice were randomly assigned to two groups; normoxia $\left(\mathrm{N}, 21 \% \mathrm{O}_{2}\right)$ or hypoxia

$177\left(\mathrm{H}, 14 \% \mathrm{O}_{2}\right)$. For hypoxic incubations, mice were transferred to an environmental

178 chamber (Coy $\mathrm{O}_{2}$ In Vivo Glove Box, Coy Laboratory Products, Grass Lake, MI) at

179 gestational day (GD) 6 where they were subjected to $14 \%$ oxygen. Levels of humidity

$180(60 \%), \mathrm{CO}_{2}(<1 \%)$ and temperature $\left(22^{\circ} \mathrm{C}\right)$ were controlled throughout chamber

181 incubation. Maternal food intake and water intake was monitored at regular intervals

182 throughout the procedure, and maternal body weight was measured before and after

183 chamber incubation. Mice were removed from the chamber at GD 18 and allowed to

184 litter in normoxia (GD $21 \pm 1$ day). Litters were culled down to six ( 3 males and 3

185 females) to assure standardized maternal care and feeding. Sexing was done by visual

186 determination of the presence or absence of dark pigmentation on the perineum ${ }^{28,29}$.

187 Offspring were weaned from the mother at 3 weeks of age, and group housed in normal

188 conditions. Experiments were performed on mouse offspring aged between 25-32 189 weeks.

190 


\section{2.3. Electron microscopy}

193 We used electron microscopy to assess left ventricular mitochondrial morphology in 194 adult mice aged between 30-32 weeks. 5 images were analyzed from each animal $(n=2$ 195 males and 2 females from normoxic pregnancies, and $n=5$ males and 5 females from 196 hypoxic pregnancies). In brief, hearts were removed from the animal, the atria were 197 discarded, and the ventricles were cut longitudinally to separate the left and right 198 chambers. A section of the left ventricular free wall was isolated and $2 \mathrm{~mm}$ horizontal 199 slices were taken from the mid myocardial layer; care was taken to isolate the same 200 area from each animal. The slices were immediately fixed by immersion with $4 \%$ 201 formaldehyde and $2.5 \%$ glutaraldehyde in $0.1 \mathrm{M}$ HEPES. The tissue was then removed 202 from the fixation solution and prepared according to the Elisman protocol ${ }^{30}$. Briefly, the 203 tissue was stained with heavy metals, dehydrated (stepwise), infiltrated with Taab 812 204 Hard Resin (stepwise), embedded in silicon wells and finally polymerized at $60^{\circ} \mathrm{C}$ for 24 205 hours ${ }^{31}$. A single block was randomly selected from a bag and muscle orientation was 206 determined in semithin sections. Ultrathin sections (up to $1 \mathrm{~mm}^{2}$, Reichert Ultracut 207 ultramicrotome) were cut longitudinally in relation to the muscle fibres. Samples were 208 then attached to 200 mesh copper EM grids and imaged at $\times 890$ magnification with a 209 FEI Tecnai 12 Biotwin microscope at $80 \mathrm{kV}$ acceleration voltage with a Gatan Orius 210 SC1000 CCD camera. A grid was randomly selected, and squares were given 211 numerical values. A random number generator was used to select 5 grid squares per 212 animal and the images were analyzed using the free hand tool in Image $\mathrm{J}$ (version 1.52k, 213 National Institute of Health, MD). Mitochondria were traced and total mitochondrial area 214 was expressed relative to the total area of the cell (students were blinded to the 215 experimental sample). 
217 2.4. Mitochondrial function

218 Mitochondrial function was investigated in male ( $\mathrm{n}=7$ normoxic and 9 hypoxic) and 219 female ( $\mathrm{n}=7$ normoxic and 8 hypoxic) offspring aged between 26-31 weeks.

\section{2.4.1. Mitochondrial oxygen consumption and $\mathrm{H}_{2} \mathrm{O}_{2}$ production}

222 Mitochondrial respiration was assessed by high-resolution respirometry using an 223 Oroboros Oxygraph-2k (Oroboros Instruments, Innsbruck, Austria) coupled to a 224 fluorescent LED2-Module, allowing simultaneous measurement of $\mathrm{O}_{2}$ consumption and $225 \mathrm{H}_{2} \mathrm{O}_{2}$ production, respectively. Mice were killed by cervical dislocation and the heart was 226 immediately excised. $\sim 50 \mathrm{mg}$ of fresh left ventricular tissue was homogenized (IKA Ultra227 turrax T25) in mitochondrial respiration media (MiR05; containing: EGTA 0.5mM, $\mathrm{MgCl}_{2}$ $2283 \mathrm{mM}$, K-MES $60 \mathrm{mM}, \mathrm{KH}_{2} \mathrm{PO}_{4} 10 \mathrm{mM}$, HEPES $20 \mathrm{mM}$, sucrose $110 \mathrm{mM}$ and $1 \% \mathrm{BSA}$ ). $2290.16 \pm 0.04 \mathrm{mg}$ of homogenised tissue was injected into each of the two chambers of the 230 Oxygraph-2K for measurement of mitochondrial respiration and $\mathrm{H}_{2} \mathrm{O}_{2}$ production. The 231 rest of the homogenate was frozen at $-80^{\circ} \mathrm{C}$ for analysis of enzymatic function (see 232 section 2.4.2). To measure $\mathrm{H}_{2} \mathrm{O}_{2}$ production, $10 \mu \mathrm{M}$ Amplex® UltraRed and $1 \mathrm{U} / \mathrm{ml}$ 233 horseradish peroxidase (HRP) were added to each chamber. Amplex® UltraRed 234 oxidizes in the presence of $\mathrm{H}_{2} \mathrm{O}_{2}$ and forms resorufin, using HRP as a catalyst. Amplex® 235 UltraRed was excited at $563 \mathrm{~nm}$ and emission was read at $587 \mathrm{~nm}$. $5 \mathrm{U} / \mathrm{ml}$ superoxide 236 dismutase (SOD) was also added to the chambers to convert any extramitochondrial 237 superoxide $\left(\mathrm{O}_{2}^{-}\right)$to $\mathrm{H}_{2} \mathrm{O}_{2}$.

239 Substrate inhibitor titration protocols (SUIT protocols) were designed according to Pesta 240 et al ${ }^{32}$ (see Fig. 1). Firstly, pyruvate ( $\left.5 \mathrm{mM}\right)$, malate $(2 \mathrm{mM})$ and glutamate (10mM) were 241 added to achieve LEAK respiratory state with complex I (Cl) substrates in the absence of 
242 adenylates $\left(\right.$ LEAK $\left._{\mathrm{CI}}\right)$. When oxygen consumption was stable, saturating ADP $(5 \mathrm{mM})$ was

243 injected to activate oxidative phosphorylation with $\mathrm{Cl}$ substrates $\left(\mathrm{OXPHOS}_{\mathrm{CI}}\right)$. Succinate

$244(10 \mathrm{mM})$ was then added to assess the additive effects of complex II (CII) substrates on

245 oxidative phosphorylation (OXPHOS $\left.\mathrm{Cl}_{\mathrm{C}} \mathrm{CII}\right)$.

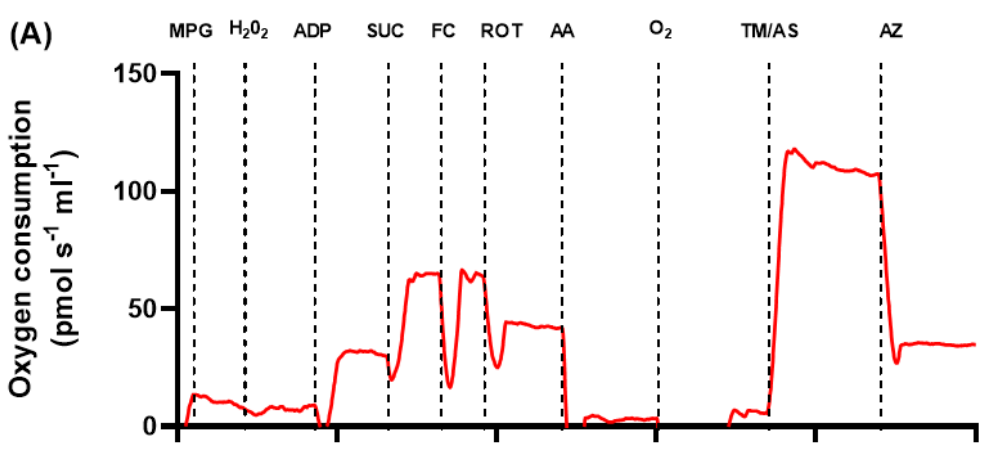

(B)

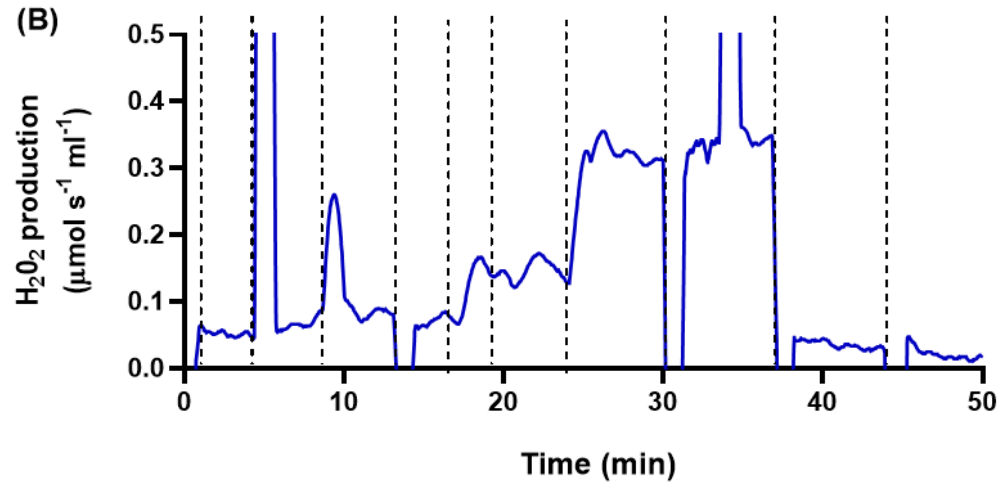

260 Figure 1: Original trace of simultaneous measurement of mitochondrial 261 oxygen consumption and $\mathrm{H}_{2} \mathrm{O}_{2}$ production in mouse ventricular homogenate.

262 Data is from a male offspring from a hypoxic pregnancy. Ventricular homogenate $263\left(0.1 \mathrm{mg} \mathrm{ml}^{-1}\right)$ was added to the chamber and a range of substrates and inhibitors 264 were injected to investigate the electron transport chain (see methods section for 265 details). Abbreviations; MPG, malate pyruvate and glutamate; ADP, adenosine 266 diphosphate; SUC, succinate; FC, Carbonyl cyanide-4267 (trifluoromethoxy)phenylhydrazone (FCCP); ROT, rotenone; $A A$, antimycin-A; $O_{2}$, 
268 the chamber was opened for reoxygenation; TM/AS, N,N,N,N-tetramethyl-p269 phenylenediamine (TMPD) and ascorbate; $A Z$, azide.

270

271 To uncouple mitochondria and assess maximum electron transfer capacity with $\mathrm{Cl}+\mathrm{Cll}$ 272 substrates ( $\mathrm{ET}_{\mathrm{Cl}+\mathrm{CII}}$ ), carbonyl cyanide-4-(trifluoromethoxy)phenylhydrazone (FCCP) was 273 titrated in steps to a final concentration of $0.1-0.3 \mu \mathrm{M}$. Next, the $\mathrm{Cl}$ inhibitor rotenone $274(0.5 \mu \mathrm{M})$ was added to assess $\mathrm{ET}_{\mathrm{Cl}+\mathrm{CII}}$ with $\mathrm{Cll}$ substrates only. To block the ET-pathway 275 and assess residual non-mitochondrial oxygen consumption (ROX), the complex III (CIII) 276 inhibitor, antimycin A, was added $(2.5 \mu \mathrm{M})$. To assess complex IV (CIV) activity in 277 isolation, the electron donor $\mathrm{N}, \mathrm{N}, \mathrm{N}^{\prime}, \mathrm{N}^{\prime}$-tetramethyl-p-phenylenediamine (TMPD, $0.5 \mathrm{mM}$ ) 278 was added in combination with ascorbate $(2 \mathrm{mM})$ to avoid autooxidation of TMPD. 279 Lastly, the CIV inhibitor sodium azide $(50 \mathrm{mM})$ was added to assess background non280 mitochondrial oxygen consumption from the addition of TMPD.

281

282 A separate protocol was used to assess the impact of $\mathrm{CIV}$ inhibition on $\mathrm{H}_{2} \mathrm{O}_{2}$ production 283 in control adult mice $(n=4)$. Ventricular homogenates were incubated with substrates to 284 achieve a steady-state $\mathrm{OXPHOS}_{\mathrm{Cl}+\mathrm{CII}}$ (pyruvate, malate, glutamate, succinate and ADP) 285 whilst measuring $\mathrm{H}_{2} \mathrm{O}_{2}$ (HRP, amplex red and SOD). Next, sodium azide was titrated in $2860.25 \mathrm{mM}$ steps from 0 to $1.75 \mathrm{mM}$ to selectively inhibit CIV.

288 All $\mathrm{O}_{2}$ consumption and $\mathrm{H}_{2} \mathrm{O}_{2}$ production data were normalized to a marker of 289 mitochondrial density, citrate synthase (CS) activity. When analyzing the effect of 290 sodium azide on $\mathrm{H}_{2} \mathrm{O}_{2}$ production, $\mathrm{H}_{2} \mathrm{O}_{2}$ was expressed as a percentage of the amount of $291 \mathrm{O}_{2}$ consumed $\left(\mathrm{H}_{2} \mathrm{O}_{2} / \mathrm{O}_{2}\right)$. To estimate mitochondrial efficiency of ATP production, the

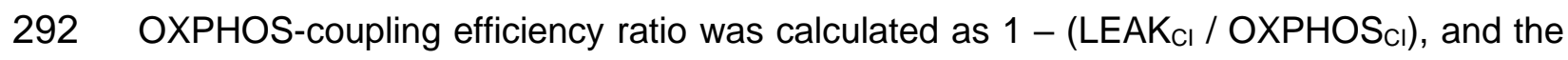
293 respiratory control ratio (RCR) was calculated as $\mathrm{OXPHOS}_{\mathrm{CI}} / \mathrm{LEAK}_{\mathrm{CI}}{ }^{33}$. 
295 2.4.2. Spectrophotometric analysis of enzymatic activities and antioxidant capacity

296 Spectrophotometric assays were undertaken on ventricular homogenates from male $(n=$ 2975 normoxic and 5 hypoxic) and female ( $n=6$ normoxic and 6 hypoxic) frozen tissue 298 samples from (aged between 25-29 weeks). Homogenates were assayed for protein 299 content, citrate synthase activity and enzymatic activity of electron transport chain 300 complexes. For all assays, absorbance was measured with a BioTek Synergy HTX 301 multimode reader (BioTek, Swindon, UK). For enzymatic assays, values are expressed 302 as maximum enzymatic activity per min divided by protein content. Protein content of 303 ventricular homogenates were assessed using the Quick start Bradford dye reagent kit 304 (Bio-Rad laboratories, Watford, UK); absorption was read at $550 \mathrm{~nm}$ at $25^{\circ} \mathrm{C}$.

305

306 Protocols for measuring $\mathrm{Cl}, \mathrm{Cll}$ and $\mathrm{ClV}$ enzyme activities, as well as citrate synthase 307 activity, were designed according to Spinazzi et al. ${ }^{34}$. In brief, approximately $20-50 \mathrm{mg}$ of 308 frozen ventricular tissue was homogenized using a FastPrep-24 ${ }^{\mathrm{TM}} 5 \mathrm{G}$ instrument (MP 309 Biomedicals, Santa Ana, CA) in solution containing 20mM TRIZMA-base, $40 \mathrm{mM} \mathrm{KCl}$, $3102 \mathrm{mM}$ EGTA and $250 \mathrm{mM}$ Sucrose $(\mathrm{pH}$ 7.4). The tissue was homogenized in two cycles of 31130 seconds with a 180 -second pause in between each trial at $4^{\circ} \mathrm{C}$. Samples were then 312 spun at $600 \mathrm{~g}$ for 10 minutes at $4^{\circ} \mathrm{C}$ and the supernatant was stored in $-80^{\circ} \mathrm{C}$ until the 313 day of the assay. Maximal enzymatic activity rate $\left(V_{\max }\right)$ was assessed over a ten-minute 314 period with a BioTek Synergy HTX multimode reader (BioTek, Swindon, UK) at $37^{\circ} \mathrm{C}$. 315 The buffer components for each individual assay are given in Supplementary Table S1. 316 
319 Protein expression of complexes in the electron transport chain was measured with

320 Western Blot in males from normoxic $(n=5)$ and hypoxic $(n=5)$ pregnancies, and

321 females from normoxic $(n=5)$ and hypoxic $(n=5)$ pregnancies, aged between 26-32

322 weeks. The protocols have been described previously in detail ${ }^{35-37}$. Following cardiac

323 excision, a $\sim 0.5 \mathrm{~cm}^{3}$ region of the left ventricular free wall was removed, snap frozen and

324 stored in liquid nitrogen until analysis. Whole homogenates ( $\sim 50 \mathrm{mg}$ starting material)

325 were prepared in RIPA buffer with protease and phosphatase inhibitors $(0.1 \mathrm{mg} / \mathrm{ml}$

326 phenylmethanesulphonylfuroide, $100 \mathrm{mmol} / \mathrm{l}$ sodium orhtovanadate, $1 \mathrm{mg} / \mathrm{ml}$ aprotonin,

$3271 \mathrm{mg} / \mathrm{ml}$ leupeptin) and protein content was determined (DC Protein Assay, BioRad,

328 UK). Proteins were separated by PAGE and transferred to nitrocellulose membranes.

$32910 \mu \mathrm{g}$ of protein was used for each sample, and membranes were blocked with $5 \%$ milk

330 in TBS-T and incubated with the primary antibody cocktail (Abcam-110413, Cambridge,

331 UK: 1:1000 concentration) and the secondary antibody IRDye® $800 \mathrm{CW} \lg _{2 \mathrm{a}}$-Specific

332 (Licor, UK: 1:20000 concentration). Membranes were visualized by chemiluminescence

333 (Syngene, UK) or IR-Dye labeled secondary antibodies (Licor, UK). As the 'classical'

334 housekeeping proteins can prove problematic with experimental treatments we opted for

335 a total protein stain to normalize for sample loading, as suggested by Li et. Al., ${ }^{38}$, and an

336 internal control to normalize between gels. Total protein transferred to the membrane

337 was determined by REVERT total protein stain (Licor, UK). Blots were repeated in

338 triplicate on separate occasions and data was averaged.

340 The antibody generated five separate bands, one for each protein corresponding to the

341 five complexes of the ETC pathway (Fig. 1 supplementary material). The value for each

342 separate protein in each sample was divided by the total value for the total protein as

343 well as the internal control. 
345 2.6. Calculations and statistics

346 Maternal BW, FI, WI are expressed as scatter plots (including all measured points) and 347 statistically compared by fitting linear regression curves to compare slopes. Offspring

348 BW is presented as scatter plots expressing means \pm SEM and compared with mixed-

349 effect analysis using maternal oxygen level as the random effect and the Tukey's

350 correction for multiple comparisons test. For CIV inhibition, a linear regression curve was

351 fitted to the data to assess if the slope was non-zero. For mitochondrial density where 352 multiple observations $(n)$ have been obtained from the same animal $(N)$, linear mixed 353 modeling (SPSS Statistics. IBM, USA) was performed thus accounting for the nested 354 (clustered) design of the experiment e.g. multiple observations from the same heart (with 355 treatment (hypoxic/normoxic), sex and animal as co-factors). All remaining data were 356 statistically analyzed using a 2-way analysis of covariance (ANCOVA), with sex and 357 intrauterine oxygen levels as independent variables, and age as a covariate (n-values 358 and $p$-values are given in the figure legends). For mitochondrial $\mathrm{H}_{2} \mathrm{O}_{2}$ production, data 359 were log-transformed to obtain a normal distribution prior to performing the ANCOVA. 360

361 3.0. Results

362 3.1. Maternal and offspring biometry

363 There were no differences in maternal body weight, water intake or food intake between 364 normoxic and hypoxic dams (Fig. 2A-C), confirming that any programmed effects in the 365 offspring are most likely due to hypoxia alone, rather than differences in maternal food 366 consumption. There were no differences in offspring body weight between hypoxic and 367 normoxic groups at any of the ages tested (Fig. 2D-E). 

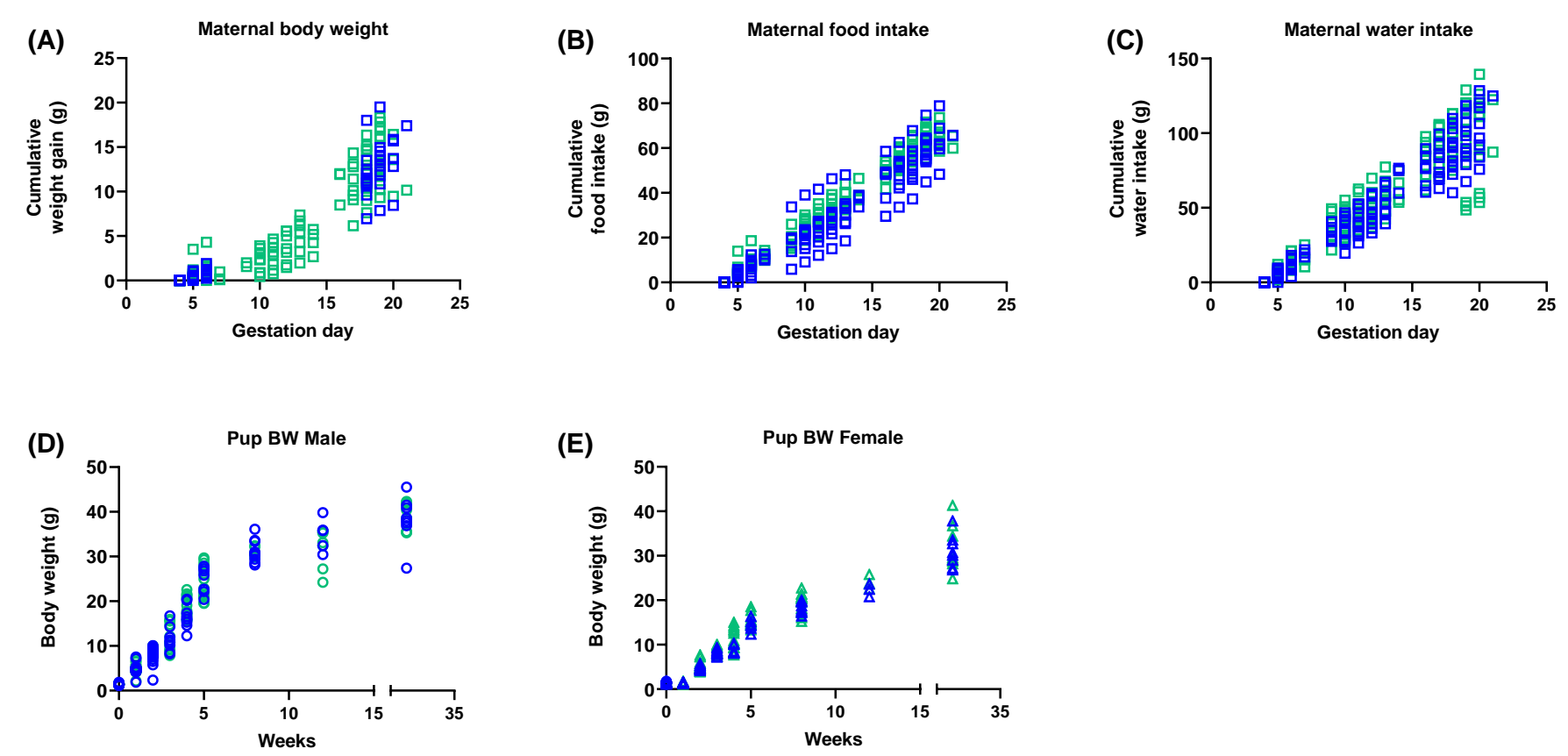

Figure 2: Effects of developmental hypoxia on maternal and fetal biometry. Maternal body weight ( $A)$, food (B) and water intake $(C)$ were monitored in normoxic (green squares, $n=16$ ) and hypoxic dams (blue squares, $n=11$ ). Due to space restrictions within the environmental chamber, the body weight of pregnant dams could not be measured during the hypoxic incubation period (gestational days 6-18). Offspring body weight was also monitored in males (D, circles) from normoxic and hypoxic pregnancies ( $n=24$ offspring from 9 pregnancies and 23 offspring from 8 pregnancies, respectively) and females ( $E$, triangles) from normoxic and hypoxic pregnancies ( $n=20$ offspring from 8 pregnancies and 20 offspring from 7 pregnancies, respectively) from birth to 15 weeks. Data are presented as scatter points for measurements of an individual animal. Statistics were run using a mixed-model effects with Tukey's correction for multiple comparisons. 
389 Mitochondrial homogenate preparations were of good quality, as attested by high 390 respiratory control ratios $(9.2+/-0.3)$ and OXPHOS-coupling efficiency ratios $(0.89+/$ 391 0.006) with complex I substrates (malate, pyruvate and glutamate). Mitochondrial 392 oxygen consumption and $\mathrm{H}_{2} \mathrm{O}_{2}$ production responded to substrates and inhibitors in the 393 expected manner ${ }^{39}$ (Fig. 1), but FCCP had modest effects on mitochondrial respiration 394 with only $9.3 \%$ of preparations responding positively. In the other preparations, even 395 very low concentrations of FCCP $(<0.1 \mu \mathrm{M})$ had no effect on oxygen consumption or 396 caused a small inhibition (see Fig. 1A), suggesting that mouse cardiac homogenates are 397 already operating at or near their theoretical maximum rate of oxygen consumption.

398

399 In male mice, developmental hypoxia reduced mitochondrial oxygen consumption in the 400 Leak $_{\mathrm{Cl}}$ and $\mathrm{OXPHOS}_{\mathrm{Cl}}$ states, as well as flux through CIV alone (Fig. 3, circular 401 symbols). In contrast, developmental hypoxia increased mitochondrial oxygen 402 consumption in female mice in all states, except for LEAK (Fig. 3, triangular symbols). 403 Within the normoxic group, male mitochondrial oxygen consumption was higher than 404 females in all respiratory states (Fig. 3, green symbols), but there were no sex405 dependent differences in the hypoxic group (Fig. 3, blue symbols). There was no effect 406 of treatment or gender on the OXPHOS-coupling efficiency ratio (Fig. 3F) or the RCR 407 (data not shown). 
414

415

416

417

418

419

420

421

422

423

424

425

426

427
(A)
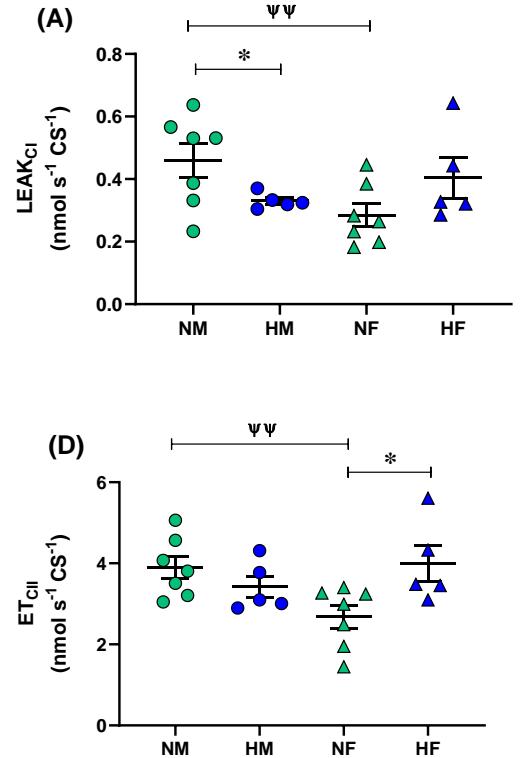
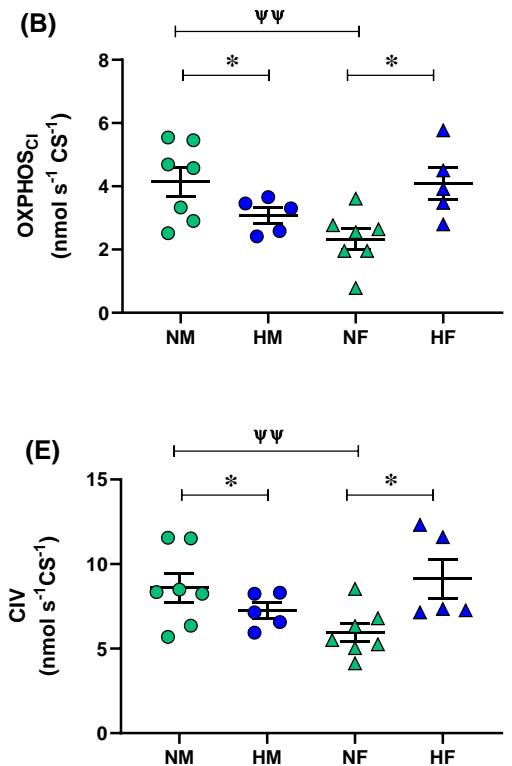
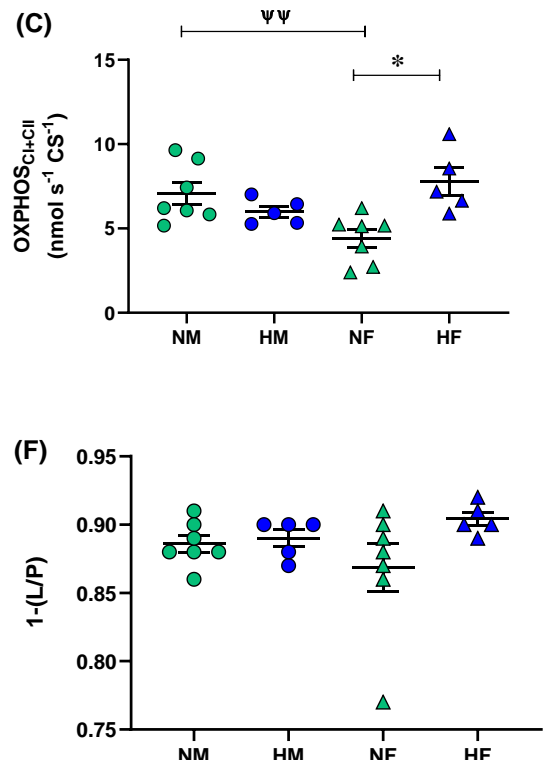

Figure 3: Effect of developmental hypoxia on mitochondrial oxygen consumption. Mitochondrial oxygen consumption was measured in males from normoxic (green circles, $n=6-7$ ) and hypoxic (blue circles, $n=8-9$ ) pregnancies, and females from normoxic (green triangles, $n=7$ ) and hypoxic (blue triangles, $n=8$ ) pregnancies. Each panel represents a different respiratory state; (A) Leak respiration with substrates for complex I (LEAKCI), (B) oxidative phosphorylation with substrates for complex I (OXPHOS $\left.C_{C}\right)$,

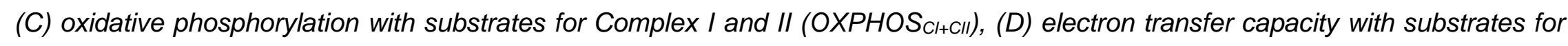
Complex II ( $E T_{C I I)}$, (E) electron donation to Complex IV (CIV) and (F) OXPHOS-coupling efficiency ratios (1-(L/P)). Data are mean \pm SEM. Asterix denotes a difference between mice from normoxic and hypoxic pregnancies, and $\Psi$ denotes a difference between male and female mice (Two-way ANCOVA, one symbol $=p<0.05$, two symbols $=p<0.01$ ). 
429 In male mice, developmental hypoxia increased $\mathrm{H}_{2} \mathrm{O}_{2}$ production when it was measured 430 in the LEAK ${ }_{C I}$ state and when CIII was inhibited with antimycin A (Fig. 4A and F, circular 431 symbols). In contrast, females from hypoxic pregnancies produced less $\mathrm{H}_{2} \mathrm{O}_{2}$ compared 432 to their normoxic counterparts in the OXPHOS ${ }_{\mathrm{Cl}+\mathrm{Cll}}$ state, and in the ET states (Fig. 4, 433 triangular symbols). With regard to sex-dependent differences, normoxic male $\mathrm{H}_{2} \mathrm{O}_{2}$ 434 production was higher than normoxic females in all respiratory states (Fig. 4, green 435 symbols), but there were no differences in $\mathrm{H}_{2} \mathrm{O}_{2}$ production between hypoxic males and 436 hypoxic females (Fig. 4, blue symbols).

438 Having discovered differences in mitochondrial oxygen consumption and $\mathrm{H}_{2} \mathrm{O}_{2}$ production 439 between treatment groups, we next sought to determine the underlying mechanisms. In 440 principle, changes in CIV oxygen consumption (Fig. 3E) may account for differences in 441 basal $\mathrm{H}_{2} \mathrm{O}_{2}$ production between experimental groups. To explore this possibility, we 442 performed experiments in control adult mice where we partially inhibited CIV with sodium 443 azide and simultaneously measured $\mathrm{H}_{2} \mathrm{O}_{2}$ production (Fig. 5). We found that a dose444 dependent inhibition of CIV caused a stepwise increase in $\mathrm{H}_{2} \mathrm{O}_{2}$ production. 

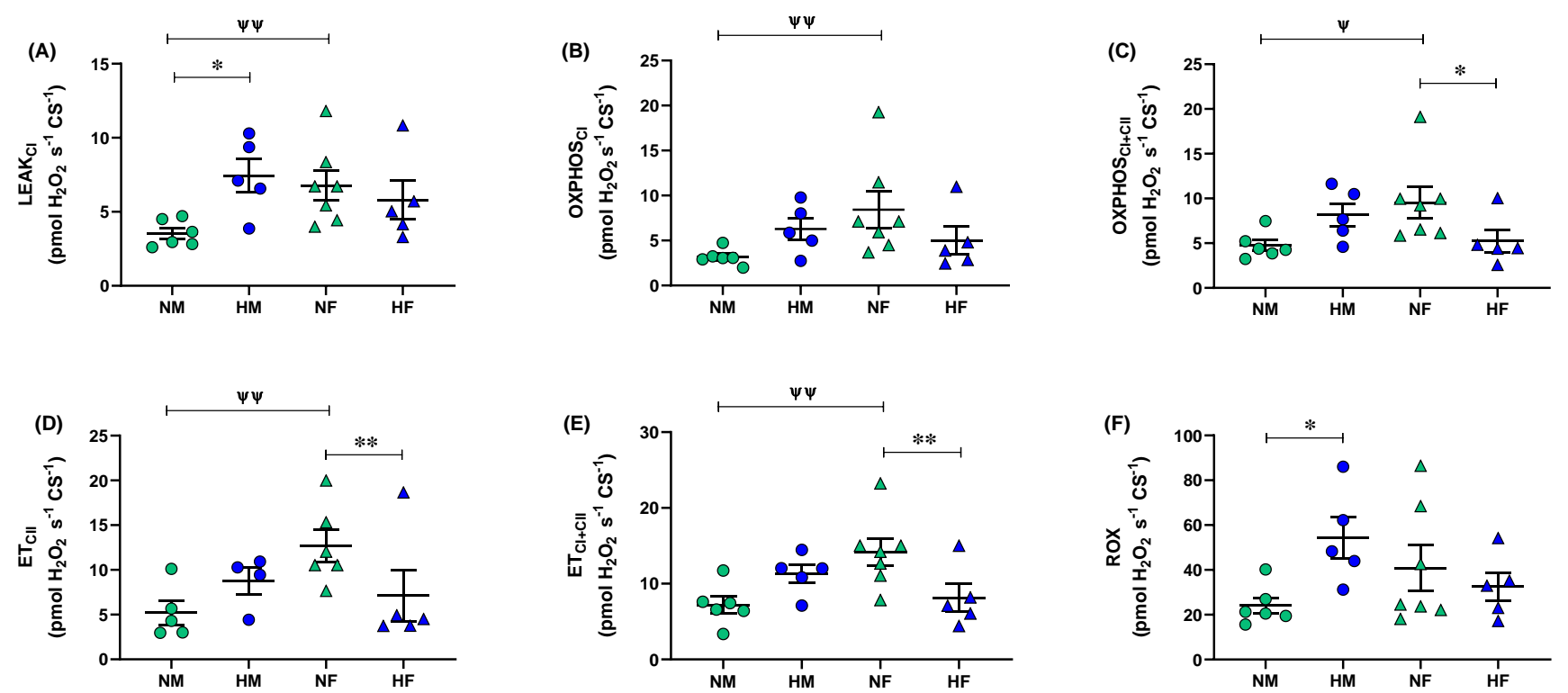

463 Figure 4: Effects of developmental hypoxia on mitochondrial $\mathrm{H}_{2} \mathrm{O}_{2}$ production. $\mathrm{H}_{2} \mathrm{O}_{2}$ production was measured in males from

464 normoxic (green circles, $n=5$ ) and hypoxic (blue circles, $n=5$ ) pregnancies, and females from normoxic (green triangles, $n=5$ ) and 465 hypoxic (blue triangles, $n=6$ ) pregnancies. Each panel represents a different respiratory state; $(A)$ Leak respiration with substrates 466 for complex I (LEAK $C_{C}$ ), (B) oxidative phosphorylation with substrates for complex I (OXPHOS ${ }_{C I}$ ), (C) oxidative phosphorylation with 467 substrates for complex I and II (OXPHOS ${ }_{C I+C I I)}$, (D) electron transfer capacity with substrates for complex II (ET $T_{C I I)}$, (E) electron 468 transfer capacity with substrates for complex $I+I I\left(E T_{C I+C I I)}\right)$ and $(F)$ residual oxygen consumption $(R O X)$ in the presence of antimycin 469 A. Data are mean \pm SEM. Asterix denotes a statistically significant difference between mice from normoxic and hypoxic

470 pregnancies, and $\Psi$ denotes a difference between male and female mice (Two-way ANCOVA, one symbol $=p<0.05$, two symbols $=$ $471 p<0.01)$. 

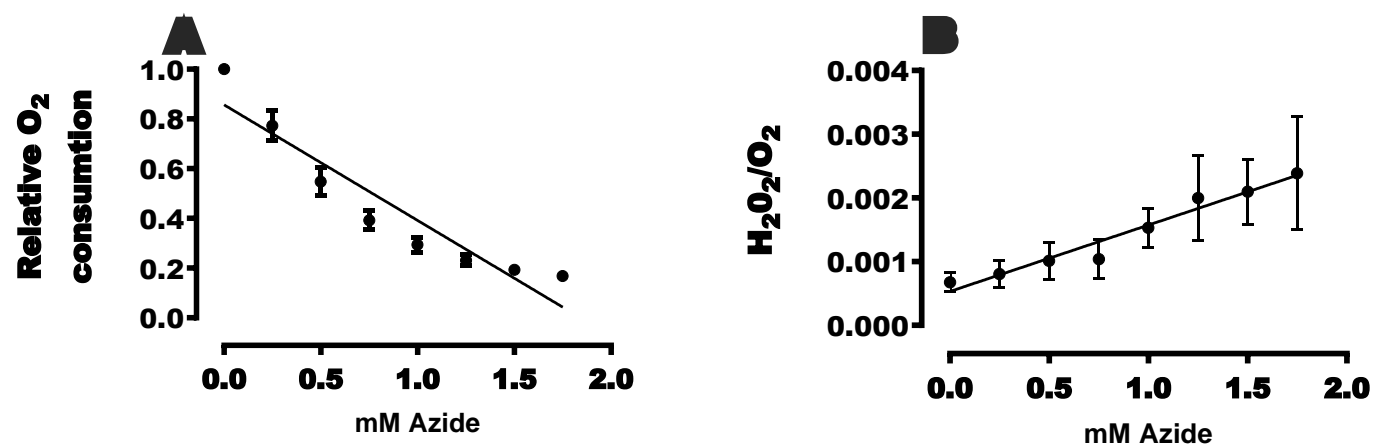

Figure 5: Relationship between complex IV activity and $\mathrm{H}_{2} \mathrm{O}_{2}$ production. presented as mean \pm SEM, $n=4$ adult females from normoxic pregnancies in both panels. A linear regression curve was fitted to the data and was found to be significantly different from zero.

491

\subsection{Offspring mitochondrial morphology}

493 Differences in mitochondrial oxygen consumption and $\mathrm{H}_{2} \mathrm{O}_{2}$ production between 494 experimental groups may also be explained by variable mitochondrial densities. 495 Therefore, we investigated mitochondrial morphology with electron microscopy and 496 measured the enzymatic activity of a common marker for mitochondrial content, citrate 497 synthase. Developmental hypoxia had no effect on mitochondrial density or citrate 498 synthase activity in any of the treatment groups (Fig. 6) and there were no differences 499 between genders.

500

501

502

503

504

505 

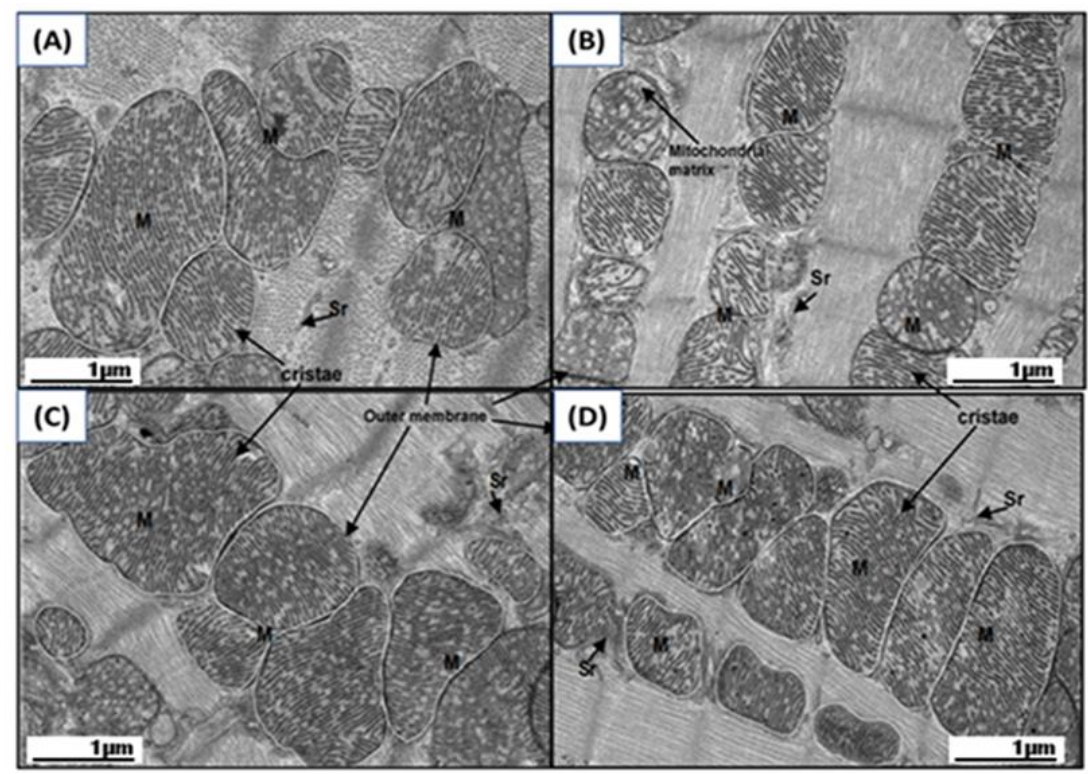

516
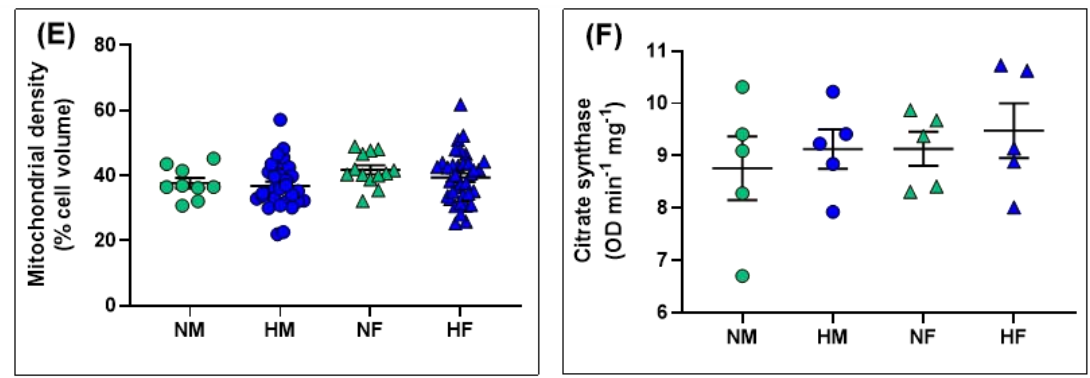

521

Figure 6: Effects of developmental hypoxia on mitochondrial density and citrate synthase activity. Transmission electron microscope images of left ventricular mitochondria in male offspring from normoxic $(A, N=2)$ and hypoxic $(B, N=5)$ pregnancies, and female offspring from normoxic $(C, N=2)$ and hypoxic $(D, N=5)$ pregnancies. Labels in image represent; M: mitochondria; Sr: sarcoplasmic reticulum; Scale bar: 1 uM. Panel E-F show mean data \pm SEM for mitochondrial density ( $n=5$ images per mouse) and citrate synthase activity ( $n=$ 5 offspring per group), respectively. For mitochondrial density, linear mixed modeling was performed to account for the nested (clustered) design of the experiment e.g. multiple observations from the same animal. For citrate synthase activity, a 2-way ANCOVA was performed. No statistical differences were found between any of the experimental groups. 
537 Next, we investigated the possibility that differences in mitochondrial oxygen

538 consumption and $\mathrm{H}_{2} \mathrm{O}_{2}$ production were underlined by variable enzymatic activities of

539 complexes in the electron transport chain. We found that male offspring from hypoxic

540 pregnancies had higher $\mathrm{Cl}$ enzymatic activity (Fig. 7A), compared to their normoxic

541 counterparts, and females from hypoxic pregnancies had lower Cll activity (Fig. 7B) and

542 higher CIV activity (Fig. 7C), compared to their normoxic counterparts.

543

544

545

546

547
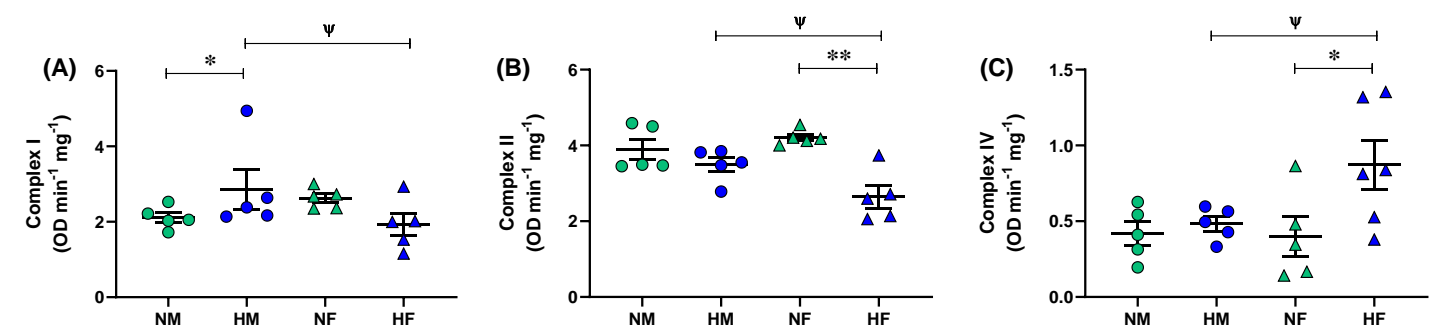

548

549

550

551

552

553

554

555

556

Figure 7: Effects of developmental hypoxia on the activity of key

557

mitochondrial enzymes. Enzymatic activity of complex I $(A)$, complex II $(B)$ and complex IV (C) were measured in males from normoxic (green circles, $n=5$ ) and hypoxic (blue circles, $n=5$ ) pregnancies, and females from normoxic (green triangles, $n=5$ ) and hypoxic (blue triangles, $n=6$ ) pregnancies. Data are

With regards to gender-specific effects, there were no differences in enzymatic activity between males and females in offspring from normoxic pregnancies, but females from

560 hypoxic pregnancies had lower $\mathrm{Cl}$ and $\mathrm{Cll}$ activity, and higher CIV activity, compared to 561 their male counterparts (Fig. 7A-C).

562

563 
564 3.6. Offspring mitochondrial complex I-V protein expression

565 Lastly, we investigated the possibility that differences we observed in mitochondrial

566 function could be explained by differential protein expression of electron transport chain

567 complexes. $\mathrm{Cl}, \mathrm{Cll}$ and $\mathrm{CIV}$ protein expression were lower in males from hypoxic

568 pregnancies, compared to their normoxic counterparts (Fig. 8A, B and D), but there were

569 no differences in the female group. Within the normoxic group, males had higher $\mathrm{Cl}, \mathrm{CII}$

570 and CIV protein expression compared to females (Fig. 8A, B and D), but there were no

571 sex-specific effects within the hypoxic group.

572

573

574

575

576

577

578

579 
580

581

582

583

584

585

586

587

588

589

590

591
(A)
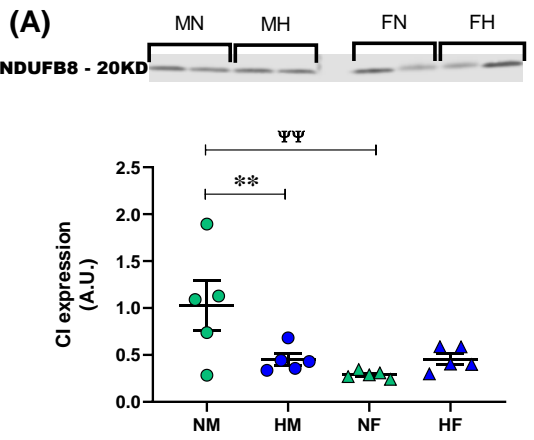

(D)
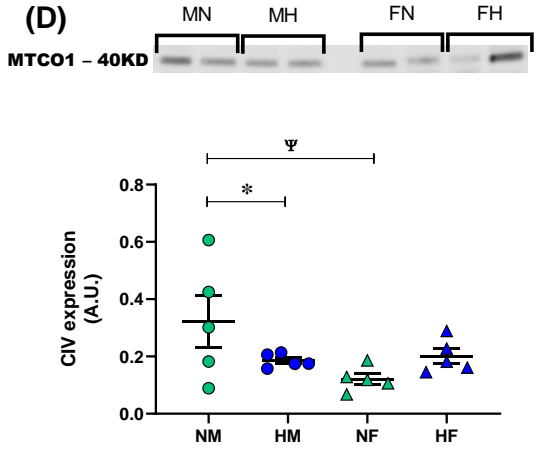
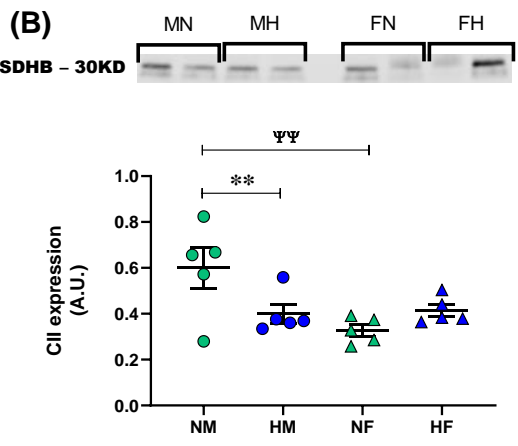

(E)

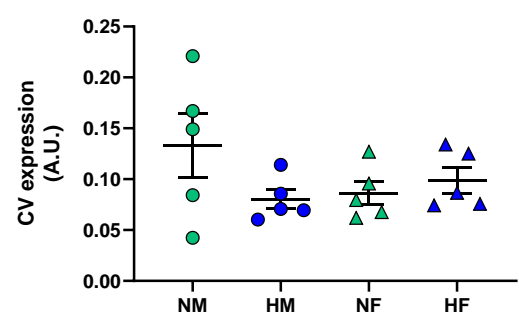

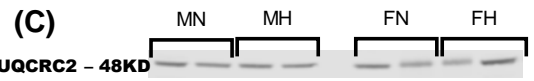

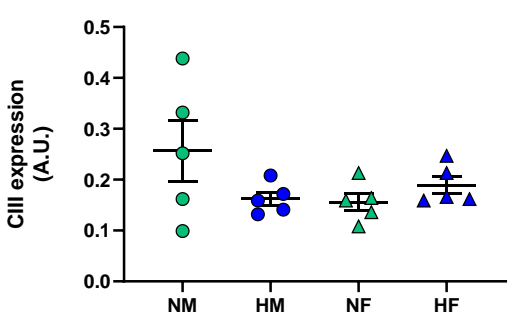

592 Figure 8: Effect of developmental hypoxia on protein expression of respiratory chain Complexes. Protein expression was

593 measured with Western Blot in males from normoxic (green circles, $n=5$ ) and hypoxic (blue circles, $n=5$ ) pregnancies, and females

594 from normoxic (green triangles, $n=5$ ) and hypoxic (blue triangles, $n=6$ ) pregnancies. Each panel represents a different complex in

595 the respiratory chain; complex I (A), II (B), III (C), IV (D) and V (E). Data are mean $\pm S E M$. Asterix denotes a difference between

596 mice from normoxic and hypoxic pregnancies, and $\Psi$ denotes a statistically significant difference between male and female mice

597 (Two-way ANCOVA, one symbol $=p<0.05$, two symbols $=p<0.01$ ). The full Blot is given in supplementary Figure $S 1$. 


\section{4.0. Discussion}

599 This is the first comprehensive study to address the effects of developmental hypoxia on 600 mitochondrial function in adult offspring. We show that male adult offspring from hypoxic 601 pregnancies possess mitochondria with a reduced respiratory capacity, increased $\mathrm{H}_{2} \mathrm{O}_{2}$ 602 production and a lower protein expression of $\mathrm{Cl}, \mathrm{Cll}$ and $\mathrm{CIV}$. In contrast, females from 603 hypoxic pregnancies had a higher respiratory capacity, greater CIV enzymatic activity, 604 and reduced $\mathrm{H}_{2} \mathrm{O}_{2}$ production, despite lower enzymatic activity of ClI. From these results, 605 we speculate that early exposure to hypoxia has long term sex-dependent effects on 606 metabolic function, which may have implications for susceptibility to cardiac disease in 607 adulthood.

608

609 4.2. Effects of developmental hypoxia on male offspring mitochondrial function

610 The first major and novel finding from this study is that male offspring from hypoxic 611 pregnancies had greater levels of basal $\mathrm{H}_{2} \mathrm{O}_{2}$ production, compared to their normoxic 612 counterparts. Previous studies have shown developmental hypoxia can cause oxidative 613 stress in fetal ${ }^{22}$ and perinatal ${ }^{40}$ mammals, but to our knowledge, this is the first study to

614 show developmental hypoxia can programme ROS levels in adulthood. It is well-known

615 that the over-production of mitochondrial ROS plays a major role in cardiac pathologies,

616 particularly $\mathrm{I} / \mathrm{R}$ injury ${ }^{41}$. Interestingly, several studies have shown developmental

617 hypoxia programmes cardiac sensitivity to $\mathrm{I} / \mathrm{R}$ injury in adult male offspring ${ }^{7,11,12}$, partly

618 due to the downregulation of protein kinase $\mathrm{C}$ epsilon (PKC $\varepsilon$ ) via DNA methylation ${ }^{13}$. In

619 addition to differential expression of $\mathrm{PKC} \varepsilon$, it is tempting to speculate that elevated basal

$620 \mathrm{H}_{2} \mathrm{O}_{2}$ production sets a functional deficit in the hearts of males from hypoxic pregnancies, 621 which may predispose them to postischemic oxidative stress. 
623 Our data provides some information on the possible mechanism underlying the elevated 624 basal $\mathrm{H}_{2} \mathrm{O}_{2}$ production in males from hypoxic pregnancies. $\mathrm{H}_{2} \mathrm{O}_{2}$ production in hypoxic 625 males was higher than their normoxic counterparts in all respiratory states (although it 626 only reached statistical significance in the LEAK $K_{C I}$ state), and the difference was also 627 apparent in the presence of rotenone and antimycin $\mathrm{A}$, which block the $\mathrm{Cl}_{\mathrm{Q}}$ and $\mathrm{Clll}_{\mathrm{Q}}$ 628 ROS sites, respectively ${ }^{42}$. In the presence of these inhibitors, ROS production 629 commonly occurs at the $\mathrm{Clll}_{\mathrm{Q}}$ site, which is a major source of oxidative stress in the 630 postischemic heart ${ }^{43}$. Nevertheless, pharmacological inhibition of the Clll $\mathrm{Q}_{0}$ site (e.g. 631 with myxothiazol or stigmatellin) would be necessary to confirm its involvement because 632 other sites proximal to CIII can contribute to ROS production under these conditions ${ }^{42}$. 633 Furthermore, other factors may explain our results, including differential antioxidant 634 profiles. Clearly, further research is necessary to confirm the site or source of elevated $635 \mathrm{H}_{2} \mathrm{O}_{2}$ production in males from hypoxic pregnancies.

637 In addition to $\mathrm{H}_{2} \mathrm{O}_{2}$ production, developmental hypoxia significantly reduced mitochondrial 638 oxygen consumption in LEAK $\mathrm{Cl}_{\mathrm{Cl}}$ and $\mathrm{OXPHOS}_{\mathrm{Cl}}$ states, as well as flux through CIV alone. 639 The reduction in mitochondrial respiration was associated with a decrease in $\mathrm{Cl}, \mathrm{Cll}$ and 640 CIV protein expression, suggesting that these complexes are downregulated by 641 developmental hypoxia, leading to lower respiratory capacity. These results are in 642 agreement with data from Thompson's group ${ }^{19,20}$ that showed developmental hypoxia 643 reduces mitochondrial respiration rate and enzymatic activities of $\mathrm{Cl}$ and $\mathrm{CIV}$ in adult 644 male guinea pigs from hypoxic pregnancies. A reduction in mitochondrial respiratory 645 capacity compromises ATP production, especially under situations of increased 646 metabolic demand, such as exercise or disease. Therefore, in addition to elevated basal 
$647 \mathrm{H}_{2} \mathrm{O}_{2}$ production, limitations in mitochondrial electron transport may contribute to cardiac 648 dysfunction and sensitivity to $\mathrm{l} / \mathrm{R}$ in males from hypoxic pregnancies.

650 The mitochondrial phenotype in males from hypoxic pregnancies may be explained by 651 several factors. It is possible that developmental hypoxia directly altered fetal 652 mitochondrial function and the phenotype persisted into adulthood. Indeed, several 653 studies have shown intrauterine stress alters embryonic and fetal mitochondria, leading 654 to the overproduction of ROS and a self-reinforcing cycle of mitochondrial dysfunction 655 that persists into adulthood, and may even be inherited ${ }^{44,45}$. In support of this idea, a 656 previous study found evidence of oxidative stress in male fetal guinea pig hearts 657 exposed to $10.5 \%$ oxygen, and this was associated with a reduction in the enzymatic 658 activity of CIV ${ }^{22}$. Alternatively, developmental hypoxia may have altered fetal 659 mitochondria indirectly due to its effects on cardiac structure. The foetus initially 660 responds to hypoxia by preferentially distributing blood flow to the heart and brain, which 661 eventually leads to increased peripheral resistance and cardiac remodeling ${ }^{4-6}$. This 662 remodeling is apparent at the cellular level ${ }^{46-48}$, which may require mitochondrial 663 alterations. Lastly, mitochondrial programming by intrauterine stress could also be 664 achieved via stable epigenetic alterations to the nuclear genome ${ }^{49}$. Indeed, recent 665 studies have shown developmental hypoxia alters global DNA methylation patterns in 666 rats leading to a reprogramming of the cardiac transcriptome, with a major focus on 667 mitochondrial genes ${ }^{50}$. Therefore, the observed differences in male mitochondrial 668 protein expression in the present study may have epigenetic origins. Integrative 669 longitudinal studies that monitor the effects of developmental hypoxia on mitochondrial 670 function across the life course would help to discern between these possibilities.

\section{1}

672 4.2. Effects of developmental hypoxia on female offspring mitochondrial function 
673 The second major and novel finding from this study is that adult female offspring from

674 hypoxic pregnancies possess mitochondria with a higher respiratory capacity, increased 675 mitochondrial efficiency and lower levels of basal $\mathrm{H}_{2} \mathrm{O}_{2}$ production. These results 676 suggest developmental hypoxia programmes a mitochondrial phenotype in females that 677 can generate ATP at a higher capacity and efficiency, while limiting ROS production. To 678 our knowledge, this is the first study which has shown developmental hypoxia can 679 programme a seemingly advantageous metabolic phenotype. From this perspective, it is 680 interesting to note that several studies have shown female hearts are less affected by 681 developmental hypoxia than males ${ }^{20,51,52}$, and they also recover better from $\mathrm{I} / \mathrm{R}$ injury $6827,11,12$. In theory, a greater mitochondrial respiratory capacity and lower $\mathrm{H}_{2} \mathrm{O}_{2}$ production 683 could help to sustain ATP production and limit oxidative stress during oxygen and/or 684 nutrient deprivation. Therefore, we propose that mitochondrial adaptations programmed 685 by developmental hypoxia may protect the female heart from subsequent $\mathrm{I} / \mathrm{R}$ stress in 686 adulthood.

688 The increased respiratory capacity in females from hypoxic pregnancies was present 689 under all respiratory states (except for Leak respiration), and with substrate 690 combinations for $\mathrm{Cl}$ and $\mathrm{CII}$, as well as direct electron transfer to CIV with TMPD. 691 Therefore, one mechanistic explanation is that developmental hypoxia increased 692 mitochondrial density in female mice. However, analysis of cardiomyocyte EM images 693 found no significant differences in mitochondrial content between females from normoxic 694 or hypoxic pregnancies. While we acknowledge that our EM analysis has limitations and 695 a more robust approach should be taken to confirm these morphological findings (i.e. 696 using stereology or 3D reconstruction), we also found no differences in female citrate 697 synthase activity, which is an excellent marker of mitochondrial content ${ }^{53}$. Taken 698 together, it seems unlikely that the differences in respiratory capacity between females 
699 from normoxic and hypoxic pregnancies is related to mitochondrial content. An 700 alternative explanation is that developmental hypoxia increases CIV activity in female 701 mice. In support of this explanation, enzymatic activity of CIV was elevated in females 702 from hypoxic pregnancies, compared to their normoxic counterparts, and this was 703 associated with a trend towards higher CIV protein expression. In addition to increasing 704 respiratory capacity, enhanced complex IV activity is also known to increase 705 mitochondrial oxygen affinity which is beneficial for maintaining ATP production under 706 conditions of low oxygen availability, such as $\mathrm{I} / \mathrm{R}$ injury ${ }^{54,55}$. Therefore, it would be 707 interesting to investigate the effects of developmental hypoxia on female mitochondrial 708 oxygen kinetics.

709

710 With regard to the mechanism underlying the reduced basal $\mathrm{H}_{2} \mathrm{O}_{2}$ production, enzymatic 711 activity and protein expression of the main ROS producing complexes ( $\mathrm{Cl}$ and $\mathrm{CIII})$ were 712 not altered by developmental hypoxia in female mice. However, theoretically, the 713 increase in CIV activity in females from hypoxic pregnancies could decrease the 714 reduction of redox centers in $\mathrm{Cl}$ or $\mathrm{CIII}$, thereby reducing electron leak and ROS 715 generation from these complexes ${ }^{56}$. To explore this possibility, we performed 716 experiments in control adult mice where we partially inhibited CIV with sodium azide and 717 simultaneously measured $\mathrm{H}_{2} \mathrm{O}_{2}$ production. We found that a dose-dependent inhibition of $718 \mathrm{CIV}$ caused a stepwise increase in $\mathrm{H}_{2} \mathrm{O}_{2}$ production. This relationship has been 719 demonstrated previously in isolated cardiomyocytes from embryonic chick 720 cardiomyocytes where reduced CIV leads to enhanced ROS production ${ }^{57}$. Therefore, 721 greater CIV activity in females from hypoxic pregnancies may contribute to the observed 722 reduction in $\mathrm{H}_{2} \mathrm{O}_{2}$ production in this experimental group. Nevertheless, there are other 723 mechanistic explanations to explore, including differences in antioxidant capacity. 
725 The mechanisms underlying mitochondrial programming in female mice are likely to be

726 similar to males (discussed above), but the resultant phenotype is obviously very 727 different. These results are strongly aligned to numerous studies that have 728 demonstrated sex-dependent differences in the susceptibility of offspring to fetal stress 729 (reviewed $\mathrm{in}^{45,58,59}$ ). Identifying the underlying cause of these differences is an active 730 area of research, but recent work suggests several fundamental differences between 731 males and females may influence programming susceptibility, including; pattern of 732 development (genetic, transcriptional and morphological), growth rate, sex hormones, 733 placental plasticity, the regulation of epigenetic processes, metabolic hormones, the rate 734 of ageing and lifespan ${ }^{45,58}$. With regard to the cardiovascular system, several studies 735 have shown oestrogen plays a protective role in the programming of hypertension, while 736 testosterone is detrimental ${ }^{59}$. Given that oestrogen is also known to modulate cardiac 737 mitochondrial biogenesis, oxidative phosphorylation and ROS production ${ }^{60}$, this hormone 738 may have played a role in orchestrating the gender-specific responses in the present 739 study. In this respect, it would be interesting to repeat the experiment in ovariectomized 740 and castrated mice. There is also strong evidence that sex-dependent differences in 741 placental plasticity play a major role in cardiovascular programming ${ }^{61}$. Indeed, 742 numerous studies have shown developmental hypoxia leads to placental remodeling in 743 rodents ${ }^{62-65}$, with female placentas adapting much better than males ${ }^{66}$. Given the 744 essential role that the placenta plays in the provision of fetal nutrients, sex-dependent 745 placental remodeling may have important consequences for cardiac function and 746 metabolism. Lastly, recent work has shown that epigenetic and transcriptomic 747 signatures associated with developmental hypoxia are sex-dependent ${ }^{67}$, and 748 predominantly mitochondrial ${ }^{50}$. Therefore, the gender-specific mitochondrial protein 749 expression that we observed may represent persistent epigenetic marks. 
751 4.3. Effects of gender on mitochondrial structure and function in offspring from normoxic 752 pregnancies

753 Previous work has demonstrated substantial differences between males and females in 754 mitochondrial structure and function, but the results are highly tissue-specific. In most 755 tissues, including liver, brain, adipose and skeletal muscle, females exhibit a higher 756 respiratory capacity than males, which is often associated with greater mitochondrial 757 content ${ }^{68}$. In contrast, two studies found no differences in cardiac mitochondrial 758 respiratory capacity between male and female rats, despite a reduced mitochondrial 759 content in females ${ }^{69,70}$. Furthermore, a third study found mitochondrial respiration with 760 glutamate and malate was higher in males compared to females ${ }^{71}$. In line with this latter 761 study, we found male normoxic mice had a greater respiratory capacity than their female 762 counterparts, and this difference was present in all respiratory states and substrate 763 combinations, as well as CIV flux with TMPD. We go further to show the increase in

764 male respiratory capacity was associated with higher male $\mathrm{Cl}, \mathrm{CII}$ and $\mathrm{CIV}$ protein 765 expression, providing a mechanistic explanation for these sex-dependent differences.

767 In addition to respiratory capacity, previous work has shown sex-dependent differences

768 in cardiac ROS production. In general, female cardiomyocytes generate less ROS than 769 males at rest and after pathological stimuli, and they are also less prone to age770 dependent oxidative stress ${ }^{69,72,73}$. Some studies suggest the reduced ROS production 771 in females is due to a superior antioxidant capacity ${ }^{73}$, while others point towards 772 reduced electron leak from $\mathrm{Cl}$ and $\mathrm{CIII}{ }^{69}$. In contrast to these studies, normoxic males in 773 our study had lower basal $\mathrm{H}_{2} \mathrm{O}_{2}$ production than their female counterparts in all 774 respiratory states and substrate combinations. We cannot explain the discrepancy 775 between these studies, but factors such as species, strain and age may play a role in 776 determining sex-dependent differences. 


\section{5.0. Conclusions and future directions}

779 In conclusion, our study has shown developmental hypoxia has long-term, sex-specific

780 implications for metabolism. We speculate that these differences may have implications 781 for disease susceptibility. For example, increased basal $\mathrm{H}_{2} \mathrm{O}_{2}$ production and lower 782 respiratory capacity in males from hypoxic pregnancies may predispose mitochondria to 783 cardiac dysfunction and I/R injury, a condition that is largely driven by oxidative stress ${ }^{41}$.

784 In contrast, the greater respiratory capacity and lower $\mathrm{H}_{2} \mathrm{O}_{2}$ production in females from 785 hypoxic pregnancies may help to sustain ATP production and limit oxidative stress under 786 conditions of oxygen deprivation. These sex-specific differences in response to 787 developmental hypoxia may help to explain why females from hypoxic pregnancies are 788 less susceptible to $\mathrm{I} / \mathrm{R}$ injury, compared to their male counterparts ${ }^{11,12}$. While purely 789 speculative at this stage, the present study provides the foundation to test this

790 hypothesis and design future gender-specific metabolic therapies to prevent cardiac 791 dysfunction in offspring from hypoxic pregnancies.

792

\section{$793 \quad 6.0$ Acknowledgements}

794 This study was funded by a PhD studentship awarded to Kim Hellgren by the Medical 795 Research Council Doctoral Training Partnerships. We would like to thank Aleksandr 796 Mironov, Dr. Christian Pinali and Samantha Forbes for their help in acquiring and 797 interpreting the EM images. In addition, we would like to thank Dr. Katherine Dibb, Dr 798 Javier Iglesias-Gonzalez and Professor David Eisner for their much-valued advice during 799 the study. 


\section{Supplementary Tables and Figures}

801 Table S1: Conditions for spectrophotometric assays of respiratory chain enzymes and citrate synthase activities in

\section{2 ventricular homogenates}

\begin{tabular}{|c|c|c|c|c|}
\hline & Cl & CII & CIV & CS \\
\hline$\lambda(\mathrm{nm})$ & 600 & 600 & 550 & 412 \\
\hline Buffer & $\mathrm{KP}, 50 \mathrm{mM}$ & $\mathrm{KP}, 25 \mathrm{mM}$ & $\mathrm{KP}, 50 \mathrm{mM}$ & TRIS, 100mM \\
\hline pH & 7.50 & 7.50 & 7.5 & 8.0 \\
\hline $\begin{array}{l}\text { Substrates/electron } \\
\text { acceptors }\end{array}$ & $\begin{array}{c}\text { NADH } 100 \mu \mathrm{M} ; \text { DCPIP } 80 \mu \mathrm{M} \text {; } \\
\text { Ub-1 } 60 \mu \mathrm{M}\end{array}$ & $\begin{array}{l}\text { Succinate } \\
20 \mathrm{mM} \text {; } \\
\text { DCPIP } 80 \mu \mathrm{M} ; \text { DUB } 50 \mu \mathrm{M}\end{array}$ & $\begin{array}{l}\text { Cyt c H2; } \\
60 \mu \mathrm{M}\end{array}$ & $\begin{array}{c}\text { DTNB } 100 \mu \mathrm{M} ; \text { Ac CoA } 300 \mu \mathrm{M} ; \\
\text { Oxaloacetate } 0.5 \mathrm{mM}\end{array}$ \\
\hline Other reagent(s) & BSA $3 \mathrm{mg} \mathrm{ml}^{-1} ; \mathrm{KCN} 300 \mu \mathrm{M}$ & BSA $1 \mathrm{mg} \mathrm{ml}^{-1} ; \mathrm{KCN} 300 \mu \mathrm{M}$ & $\mathrm{KCN} 300 \mu \mathrm{M}$ & Triton X-100 0.1\%; \\
\hline Specific inhibitor & $\begin{array}{c}\text { Rotenone } \\
10 \mu \mathrm{M}\end{array}$ & $\begin{array}{c}\text { Malonate } \\
10 \mathrm{mM}\end{array}$ & $\mathrm{KCN} 300 \mu \mathrm{M}$ & - \\
\hline \multicolumn{5}{|c|}{$\begin{array}{l}\text { Abbreviations: } C I \text {, complex I; CII, complex II; CIV, complex IV; CS, citrate synthase; } \lambda \text {, selected wavelength for the assay; } \varepsilon \text {, extinction coefficient; Ac } \\
\text { CoA, acetyl coenzyme A; BSA, fatty acid-free bovine serum albumin; Cyt c } H_{2} \text {, reduced cytochrome } c \text {; DCPIP, 2,6-dichlorophenolindophenol; DUB } \\
\text { decylubiquinol; DTNB, 5,5'-dithiobis(2-nitrobenzoic acid); KCN, potassium cyanide; KP, potassium phosphate buffer; Tris, Tris buffer; Ub1, biquinone1 }\end{array}$} \\
\hline
\end{tabular}


803

804

805

806

807

808

809

810

811

812

813

814

815

816

817

818

819

\section{(A)}

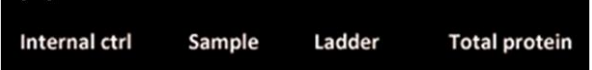

Internal ctri

Sample

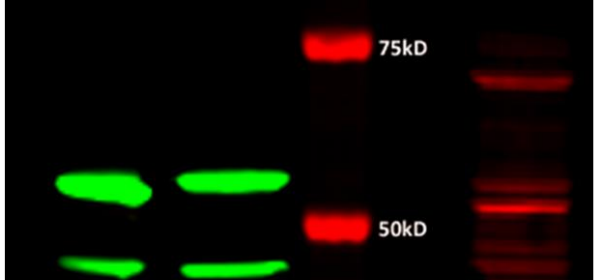

(B) Ladder

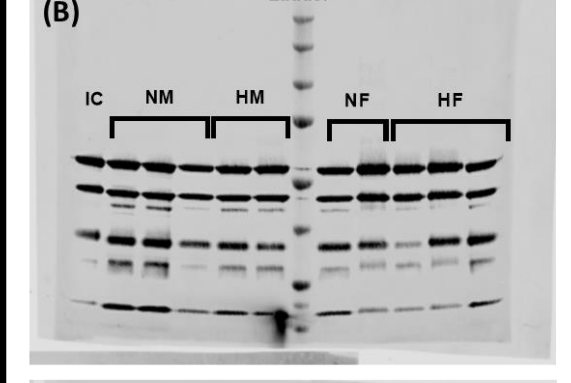

(C)

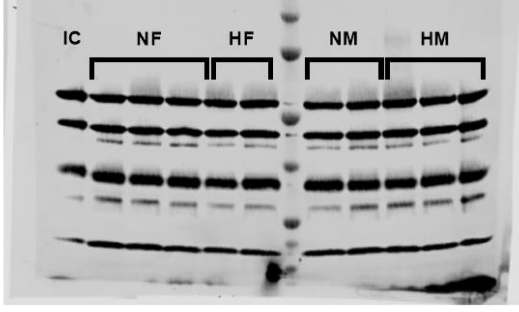

Figure S1: Representative images of Western Blots for one replicate. (A) Each gel was loaded and imaged at two wavelengths; the samples and an internal control (historical left ventricular mouse sample that was used for all blots) were loaded with the antibody and imaged at 800nm (green bands: top to bottom; complex V, complex III, complex IV, complex II, and complex I). In addition, a ladder and the total protein stain, REVERT, was imaged on the same Blot at 700nm (red bands). (B-C) Original Blots for one replicate showing sample protein expression of Complexes V, III, IV, II and I (top to bottom) in an internal control (IC), normoxic female $(N F, n=5)$, hypoxic female $(H F, n=5)$, normoxic male $(N M, n=5)$ and hypoxic male $(H M, n=5)$. Averages for three replicates are given in Figure 8 of the main manuscript. 
820

821

822

823

824

825

826

827

828

829

830

831

832

833

834

835

836

837

838

839

840

841

842

843

844

845

846

847

848

849

850

851

852

853

854

855

856

857

858

859

860

861

862

863

864

\section{References}

1. Dagenais, G.R., et al. Variations in common diseases, hospital admissions, and deaths in middle-aged adults in 21 countries from five continents (PURE): a prospective cohort study. The Lancet.

2. Barker, D.J.P., Osmond, C., Winter, P.D., Margetts, B. \& Simmonds, S.J. WEIGHT IN INFANCY AND DEATH FROM ISCHAEMIC HEART DISEASE. The Lancet 334, 577-580 (1989).

3. Hutter, D., Kingdom, J. \& Jaeggi, E. Causes and Mechanisms of Intrauterine Hypoxia and Its Impact on the Fetal Cardiovascular System: A Review. International Journal of Pediatrics 2010, 9 (2010).

4. Giussani, D.A. The fetal brain sparing response to hypoxia: physiological mechanisms. The Journal of physiology 594, 1215-1230 (2016).

5. Cohn, H.E., Sacks, E.J., Heymann, M.A. \& Rudolph, A.M. Cardiovascular responses to hypoxemia and acidemia in fetal lambs. Am J Obstet Gynecol 120, 817-824 (1974).

6. Giussani, D.A., Spencer, J.A., Moore, P.J., Bennet, L. \& Hanson, M.A. Afferent and efferent components of the cardiovascular reflex responses to acute hypoxia in term fetal sheep. The Journal of physiology 461, 431449 (1993).

7. Niu, Y., et al. Maternal Allopurinol Prevents Cardiac Dysfunction in Adult Male Offspring Programmed by Chronic Hypoxia During Pregnancy. Hypertension 72, 971-978 (2018).

8. Giussani, D.A., et al. Developmental Programming of Cardiovascular Dysfunction by Prenatal Hypoxia and Oxidative Stress. PloS one 7, e31017 (2012).

9. Zhang, L. Prenatal Hypoxia and Cardiac Programming. Journal of the

Society for Gynecologic Investigation 12, 2-13 (2005).

10. Rueda-Clausen, C.F., Morton, J.S., Lopaschuk, G.D. \& Davidge, S.T. Long-term effects of intrauterine growth restriction on cardiac metabolism and susceptibility to ischaemia/reperfusion. Cardiovascular research $\mathbf{9 0}$, 285-294 (2011).

11. Xue, Q. \& Zhang, L. Prenatal Hypoxia Causes a Sex-Dependent Increase in Heart Susceptibility to Ischemia and Reperfusion Injury in Adult Male Offspring: Role of Protein Kinase C $\epsilon$. Journal of Pharmacology and Experimental Therapeutics 330, 624-632 (2009).

12. Li, G., et al. Effect of Fetal Hypoxia on Heart Susceptibility to Ischemia and Reperfusion Injury in the Adult Rat. Journal of the Society for Gynecologic Investigation 10, 265-274 (2003).

13. Patterson, A.J., Chen, M., Xue, Q., Xiao, D. \& Zhang, L. Chronic prenatal hypoxia induces epigenetic programming of $\mathrm{PKC}\{$ epsilon\} gene repression in rat hearts. Circulation research 107, 365-373 (2010).

14. Shah, A., et al. Cardiovascular susceptibility to \&lt;em\&gt;in vivo\&lt;/em\&gt; ischemic myocardial injury in male and female rat offspring exposed to prenatal hypoxia. Clinical science 131, 2303 (2017). 
865

866

867

868

869

870

871

872

873

874

875

876

877

878

879

880

881

882

883

884

885

886

887

888

889

890

891

892

893

894

895

896

897

898

899

900

901

902

903

904

905

906

907

908

909

15. Beauchamp, B., et al. Undernutrition during pregnancy in mice leads to dysfunctional cardiac muscle respiration in adult offspring. Bioscience reports 35(2015).

16. Ferey, J.L.A., et al. A maternal high-fat, high-sucrose diet induces transgenerational cardiac mitochondrial dysfunction independently of maternal mitochondrial inheritance. American journal of physiology. Heart and circulatory physiology 316, H1202-h1210 (2019).

17. Larsen, T.D., et al. Diabetic Pregnancy and Maternal High-Fat Diet Impair Mitochondrial Dynamism in the Developing Fetal Rat Heart by SexSpecific Mechanisms. Int J Mol Sci 20, 3090 (2019).

18. Peng, J., et al. The detrimental effects of glucocorticoids exposure during pregnancy on offspring's cardiac functions mediated by hypermethylation of bone morphogenetic protein-4. Cell Death \& Disease 9, 834 (2018).

19. Thompson, L.P., Song, H. \& Polster, B.M. Fetal Programming and Sexual Dimorphism of Mitochondrial Protein Expression and Activity of Hearts of Prenatally Hypoxic Guinea Pig Offspring. Oxidative medicine and cellular longevity 2019, 7210249 (2019).

20. Thompson, L.P., Chen, L., Polster, B.M., Pinkas, G. \& Song, H. Prenatal hypoxia impairs cardiac mitochondrial and ventricular function in guinea pig offspring in a sex-related manner. Am J Physiol Regul Integr Comp Physiol 315, R1232-R1241 (2018).

21. Al-Hasan, Y.M., Pinkas, G.A. \& Thompson, L.P. Prenatal Hypoxia Reduces Mitochondrial Protein Levels and Cytochrome c Oxidase Activity in Offspring Guinea Pig Hearts. Reproductive Sciences 21, 883-891 (2014).

22. Al-Hasan, Y.M., et al. Chronic Hypoxia Impairs Cytochrome Oxidase Activity Via Oxidative Stress in Selected Fetal Guinea Pig Organs. Reproductive Sciences 20, 299-307 (2013).

23. McMillen, I.C. \& Robinson, J.S. Developmental origins of the metabolic syndrome: prediction, plasticity, and programming. Physiological reviews 85, 571-633 (2005).

24. Morrison, J.L. Sheep models of intrauterine growth restriction: fetal adaptations and consequences. Clinical and experimental pharmacology \& physiology 35, 730-743 (2008).

25. Allison, B.J., et al. Divergence of mechanistic pathways mediating cardiovascular aging and developmental programming of cardiovascular disease. FASEB journal : official publication of the Federation of American Societies for Experimental Biology 30, 1968-1975 (2016).

26. Kane, A.D., Herrera, E.A., Camm, E.J. \& Giussani, D.A. Vitamin C prevents intrauterine programming of in vivo cardiovascular dysfunction in the rat. Circulation journal : official journal of the Japanese Circulation Society 77, 2604-2611 (2013).

27. Giussani, D.A. \& Davidge, S.T. Developmental programming of cardiovascular disease by prenatal hypoxia. Journal of Developmental Origins of Health and Disease 4, 328-337 (2013). 
910

911

912

913

914

915

916

917

918

919

920

921

922

923

924

925

926

927

928

929

930

931

932

933

934

935

936

937

938

939

940

941

942

943

944

945

946

947

948

949

950

951

952

953

954

955
28. Wolterink-Donselaar, I.G., Meerding, J.M. \& Fernandes, C. A method for gender determination in newborn dark pigmented mice. Lab Anim (NY) 38, 35-38 (2009).

29. Deeney, S., Powers, K.N. \& Crombleholme, T.M. A comparison of sexing methods in fetal mice. Lab Anim (NY) 45, 380-384 (2016).

30. Pinali, C., Bennett, H., Davenport, J.B., Trafford, A.W. \& Kitmitto, A. Three-Dimensional Reconstruction of Cardiac Sarcoplasmic Reticulum Reveals a Continuous Network Linking Transverse-Tubules. Circulation Research 113, 1219-1230 (2013).

31. Deerinck, T.J., et al. Enhancing Serial Block-Face Scanning Electron Microscopy to Enable High Resolution 3-D Nanohistology of Cells and Tissues. Microscopy and Microanalysis 16, 1138-1139 (2010).

32. Pesta, D. \& Gnaiger, E. High-Resolution Respirometry: OXPHOS Protocols for Human Cells and Permeabilized Fibers from Small Biopsies of Human Muscle. Vol. 810 25-58 (Humana Press, Totowa, NJ, 2012).

33. Lemieux, H., et al. Impairment of mitochondrial respiratory function as an early biomarker of apoptosis induced by growth factor removal. bioRxiv, 151480 (2017).

34. Spinazzi, M., Casarin, A., Pertegato, V., Salviati, L. \& Angelini, C. Assessment of mitochondrial respiratory chain enzymatic activities on tissues and cultured cells. Nature Protocols 7, 1235 (2012).

35. Lawless, M., et al. Phosphodiesterase 5 inhibition improves contractile function and restores transverse tubule loss and catecholamine responsiveness in heart failure. Scientific Reports 9, 6801 (2019).

36. Briston, S.J., et al. Impaired beta-adrenergic responsiveness accentuates dysfunctional excitation-contraction coupling in an ovine model of tachypacing-induced heart failure. J Physiol 589, 1367-1382 (2011).

37. Caldwell, J.L., et al. Dependence of cardiac transverse tubules on the BAR domain protein amphiphysin II (BIN-1). Circulation research 115, 986-996 (2014).

38. Li, R. \& Shen, Y. An old method facing a new challenge: re-visiting housekeeping proteins as internal reference control for neuroscience research. Life Sci 92, 747-751 (2013).

39. Makrecka-Kuka, M., Krumschnabel, G. \& Gnaiger, E. High-Resolution Respirometry for Simultaneous Measurement of Oxygen and Hydrogen Peroxide Fluxes in Permeabilized Cells, Tissue Homogenate and Isolated Mitochondria. Biomolecules 5, 1319-1338 (2015).

40. Puente, B.N., et al. The oxygen-rich postnatal environment induces cardiomyocyte cell-cycle arrest through DNA damage response. Cell 157, 565-579 (2014).

41. Chouchani, E.T., et al. Ischaemic accumulation of succinate controls reperfusion injury through mitochondrial ROS. Nature 515, 431-435 (2014).

42. Brand, M.D. Mitochondrial generation of superoxide and hydrogen peroxide as the source of mitochondrial redox signaling. Free Radical Biology and Medicine 100, 14-31 (2016). 
956

957

958

959

960

961

962

963

964

965

966

967

968

969

970

971

972

973

974

975

976

977

978

979

980

981

982

983

984

985

986

987

988

989

990

991

992

993

994

995

996

997

998

999

1000

43. Chen, Y.-R. \& Zweier, J.L. Cardiac Mitochondria and Reactive Oxygen Species Generation. Circulation research 114, 524-537 (2014).

44. Gyllenhammer, L.E., Entringer, S., Buss, C. \& Wadhwa, P.D. Developmental programming of mitochondrial biology: a conceptual framework and review. Proceedings of the Royal Society B: Biological Sciences 287, 20192713 (2020).

45. Dearden, L., Bouret, S.G. \& Ozanne, S.E. Sex and gender differences in developmental programming of metabolism. Molecular Metabolism 15, 819 (2018).

46. Botting, K.J., McMillen, I.C., Forbes, H., Nyengaard, J.R. \& Morrison, J.L. Chronic hypoxemia in late gestation decreases cardiomyocyte number but does not change expression of hypoxia-responsive genes. Journal of the American Heart Association 3(2014).

47. Botting, K.J., et al. IUGR decreases cardiomyocyte endowment and alters cardiac metabolism in a sex- and cause-of-IUGR-specific manner. American Journal of Physiology-Regulatory, Integrative and Comparative Physiology 315, R48-R67 (2018).

48. Bae, S., Xiao, Y., Li, G., Casiano, C.A. \& Zhang, L. Effect of maternal chronic hypoxic exposure during gestation on apoptosis in fetal rat heart. Am J Physiol Heart Circ Physiol 285, H983-990 (2003).

49. Wadhwa, P.D., Buss, C., Entringer, S. \& Swanson, J.M. Developmental origins of health and disease: brief history of the approach and current focus on epigenetic mechanisms. Seminars in reproductive medicine $\mathbf{2 7}$, 358-368 (2009).

50. Gao, Y., et al. Multi-Omics Integration Reveals Short and Long-Term Effects of Gestational Hypoxia on the Heart Development. Cells 8, 1608 (2019).

51. Rueda-Clausen, C.F., Morton, J.S. \& Davidge, S.T. Effects of hypoxiainduced intrauterine growth restriction on cardiopulmonary structure and function during adulthood. Cardiovascular Research 81, 713-722 (2008).

52. Hemmings, D.G., Williams, S.J. \& Davidge, S.T. Increased myogenic tone in 7-month-old adult male but not female offspring from rat dams exposed to hypoxia during pregnancy. American Journal of Physiology-Heart and Circulatory Physiology 289, H674-H682 (2005).

53. Larsen, S., et al. Biomarkers of mitochondrial content in skeletal muscle of healthy young human subjects. The Journal of physiology 590, 3349-3360 (2012).

54. Bienfait, H.F., Jacobs, J.M. \& Slater, E.C. Mitochondrial oxygen affinity as a function of redox and phosphate potentials. Biochimica et biophysica acta 376, 446-457 (1975).

55. Gnaiger, E. Bioenergetics at low oxygen: dependence of respiration and phosphorylation on oxygen and adenosine diphosphate supply. Respiration Physiology 128, 277-297 (2001).

56. Dawson, T.L., Gores, G.J., Nieminen, A.L., Herman, B. \& Lemasters, J.J. Mitochondria as a source of reactive oxygen species during reductive 
1001

1002

1003

1004

1005

1006

1007

1008

1009

1010

1011

1012

1013

1014

1015

1016

1017

1018

1019

1020

1021

1022

1023

1024

1025

1026

1027

1028

1029

1030

1031

1032

1033

1034

1035

1036

1037

1038

1039

1040

1041

1042

1043

1044

1045

1046

stress in rat hepatocytes. The American journal of physiology 264, C961967 (1993).

57. Duranteau, J., Chandel, N.S., Kulisz, A., Shao, Z. \& Schumacker, P.T. Intracellular signaling by reactive oxygen species during hypoxia in cardiomyocytes. J Biol Chem 273, 11619-11624 (1998).

58. Aiken, C.E. \& Ozanne, S.E. Sex differences in developmental programming models. Reproduction (Cambridge, England) 145, R1-13 (2013).

59. Intapad, S., Ojeda, N.B., Dasinger, J.H. \& Alexander, B.T. Sex Differences in the Developmental Origins of Cardiovascular Disease. Physiology 29, 122-132 (2014).

60. Ventura-Clapier, R., Piquereau, J., Veksler, V. \& Garnier, A. Estrogens, Estrogen Receptors Effects on Cardiac and Skeletal Muscle Mitochondria. Front Endocrinol (Lausanne) 10, 557-557 (2019).

61. Thornburg, K.L., O'Tierney, P.F. \& Louey, S. Review: The placenta is a programming agent for cardiovascular disease. Placenta 31 Suppl, S54S59 (2010).

62. Fisher, J.J., Bartho, L.A., Perkins, A.V. \& Holland, O.J. Placental mitochondria and reactive oxygen species in the physiology and pathophysiology of pregnancy. Clinical and Experimental Pharmacology and Physiology 47, 176-184 (2020).

63. Nuzzo, A.M., et al. Placental Adaptation to Early-Onset Hypoxic Pregnancy and Mitochondria-Targeted Antioxidant Therapy in a Rodent Model. The American Journal of Pathology 188, 2704-2716 (2018).

64. Song, H., Telugu, B.P. \& Thompson, L.P. Sexual dimorphism of mitochondrial function in the hypoxic guinea pig placenta†. Biology of Reproduction 100, 208-216 (2018).

65. Sferruzzi-Perri, A.N., Higgins, J.S., Vaughan, O.R., Murray, A.J. \& Fowden, A.L. Placental mitochondria adapt developmentally and in response to hypoxia to support fetal growth. Proceedings of the National Academy of Sciences of the United States of America 116, 1621-1626 (2019).

66. Cuffe, J.S.M., et al. Mid- to late term hypoxia in the mouse alters placental morphology, glucocorticoid regulatory pathways and nutrient transporters in a sex-specific manner. The Journal of physiology 592, 3127-3141 (2014).

67. Chen, X., Zhang, L. \& Wang, C. Prenatal hypoxia-induced epigenomic and transcriptomic reprogramming in rat fetal and adult offspring hearts.

Scientific Data 6, 238 (2019).

68. Ventura-Clapier, R., et al. Mitochondria: a central target for sex differences in pathologies. Clinical science 131, 803-822 (2017).

69. Colom, B., Oliver, J., Roca, P. \& Garcia-Palmer, F.J. Caloric restriction and gender modulate cardiac muscle mitochondrial $\mathrm{H} 2 \mathrm{O} 2$ production and oxidative damage. Cardiovascular research 74, 456-465 (2007).

70. Moulin, M., et al. Sexual Dimorphism of Doxorubicin-Mediated Cardiotoxicity. Circulation: Heart Failure 8, 98-108 (2015). 
1047 71. Ribeiro, R.F., et al. Sex differences in the regulation of spatially distinct cardiac mitochondrial subpopulations. Mol Cell Biochem 419, 41-51

1049

1050

1051

1052

1053

1054

1055

1056 (2016).

72. Lagranha, C.J., Deschamps, A., Aponte, A., Steenbergen, C. \& Murphy, E. Sex differences in the phosphorylation of mitochondrial proteins result in reduced production of reactive oxygen species and cardioprotection in females. Circulation research 106, 1681-1691 (2010).

73. Kander, M.C., Cui, Y. \& Liu, Z. Gender difference in oxidative stress: a new look at the mechanisms for cardiovascular diseases. J Cell Mol Med 21, 1024-1032 (2017). 